\title{
Technology Licensing Strategy for Network Product in a Service Industry
}

\author{
Xianpei Hong, ${ }^{1}$ Dan $\mathrm{Zhao}^{2}$ Haiqing Hu, ${ }^{3}$ and Shuang Song ${ }^{4}$ \\ ${ }^{1}$ College of Economics and Management, Huazhong Agricultural University, Wuhan 430070, China \\ ${ }^{2}$ School of Management, Henan University of Science and Technology, Luoyang 471023, China \\ ${ }^{3}$ School of Business, Shandong Yingcai University, Jinan 250104, China \\ ${ }^{4}$ College of Science, Tianjin University of Technology, Tianjin 300384, China
}

Correspondence should be addressed to Dan Zhao; zhaodan911@126.com

Received 15 August 2014; Revised 15 October 2014; Accepted 2 November 2014

Academic Editor: Bin Shen

Copyright (C) 2015 Xianpei Hong et al. This is an open access article distributed under the Creative Commons Attribution License, which permits unrestricted use, distribution, and reproduction in any medium, provided the original work is properly cited.

\begin{abstract}
Technology licensing has gained significant attention in literature and practice as a rapid and effective way to improve firm's capability of technology innovation. In this paper, we investigate a duopolistic service provider competition market, where service providers develop and sell a kind of network product. In this setting, we analyze the innovating service provider's four licensing strategies: no licensing, fixed fee licensing, royalty licensing, and two-part tariff licensing. The literature suggests that when the network products can be completely substituted, two-part tariff licensing is the optimal strategy of the innovating service provider. We find that when the network products cannot be completely substituted, two-part tariff licensing is not always optimal. The degree of the product differentiation, the intensity of the network effects, and the R\&D cost of the potential licensee play a key role in determining the innovating service provider's optimal licensing strategies.
\end{abstract}

\section{Introduction}

Technology licensing not only is an important form of technology transfer under patent system but also is regarded as a crucial way to gain innovation profits through technology market for patent holder. With the rapid development of economic globalization and new technologies, technology licensing is becoming more and more common, especially in some technology-intensive industries, such as IBM, gaining a profit of $\$ 1.3$ billion (10\% of its pretax profits) through licensing the technology in 2000, and Texas Instruments which gets more than $\$ 40$ million annually from patent licensing [1].

Technology licensing is regarded as the fastest and most effective way to improve the firms' technology innovation capability in addition to independent innovation [1]. The significance of technology licensing can be specified from the following two aspects. Firstly, for firms in developing countries (such as China), confronted with overall weakness of R\&D capability, a blind encouragement on innovation may lead to higher input yet less effective output, with the loss outweighs the gain. However, technology licensing may shorten the gap between abovementioned firms and those with higher R\&D efficiency in developed countries, thus preparing for further "leapfrog." Secondly, for those firms with stronger innovation capability and higher R\&D efficiency, they should ponder over how to speed up the recovery of R\&D investment to obtain higher economic profits, especially after they have patented the core technology. Technology licensing can provide a feasible way to improve the return on investment for those kinds of firms. A study by Grindley and Teece [2] addresses that major firms in high-technology industries, such as AT\&T, IBM, Texas Instruments, and Hewlett-Packard, regard the use of licensing and cross-licensing as an important part of their business strategies.

In information and telecommunication industries, many innovating service providers choose to license their technology to others to establish an industry innovation standard. As an example, Qualcomm owned the core technology of 
CDMA and LTE, but it only established an innovation-based standard in other small markets, such as Hong Kong and Korea, before it entered the market of Chinese Mainland. Qualcomm obtained tremendous development opportunity after it licensed its CDMA technology to China Unicom. Qualcomm gained a profit of 249 hundred million dollars through chip distribution and technology licensing in 2013, among which, it gained a profit of 120 hundred million dollars ( $84 \%$ of its whole profits) from China. HUAWEI and ZTE Corporation that have adopted CDMA technology pay Qualcomm a per unit charge (5\%) to access the CDMA network. That is why so many innovating firms are keening on establishing an industry standard.

Our research question arises from practice. However, in theoretical research, few address the firm's technology licensing issues for network products. For a network product, users derive added value from the presence of others in the network; the additional network value depends on the number of other users of this product [3,4]. Different from the normal products, the market for network products continues to expand with the popularity of the Internet. This phenomenon is commonly seen in reality, for example, instant communication devices such as MSN and QQ are of no value when users are too few. However, considering the increment in the number of users of these devices, their network values and consumer evaluations continuously increase. In this paper, we define the products with network effects as network products and the products without network effects as normal products.

Based on the above analysis, the purpose of this paper is to explore the following two issues by taking account of network effects of two competing firms under the situation where the innovator serves as an insider patentee, as firms produce and engage in Cournot competition. Firstly, what is the innovating firm's optimal licensing strategy in terms of fixed-fee, royalty, or two-part tariff licensing? Secondly, how do the network intensity and product heterogeneity affect the technology licensing strategy for the innovating firm?

The main findings of this paper are as follows. (1) For the innovator provider, no matter whether the market scales are large or small, and as long as the network intensity $\beta$ is large enough (e.g., $\beta \geq 0.5$ ), two-part tariff licensing strategy is the same as fixed-fee licensing strategy. However, as for the innovator provider, the optimal licensing strategy is not always two-part tariff licensing and is different from the case of normal product. In a network product market, the optimal licensing strategy of the innovator provider depends on the market conditions; to be specific, when the market scale is very small, the optimal licensing strategy is fixedfee licensing. When the market scale is medium, royalty licensing is the optimal strategy when licensee firm's R\&D cost is very high. When the market scale is very large, the optimal licensing strategy depends mainly on network intensity, product substitute levels, and licensee firm R\&D cost. (2) When network intensity is relatively small (e.g., $\beta<$ 0.5 ) and the $R \& D$ cost is very high, two-part tariff licensing is the optimal licensing strategy of the innovator provider.

The reminder of this paper is organized as follows. In Section 2, we briefly review the related research. Section 3 describes the game process and market demand. In Section 4 we analyze three licensing strategies (i.e., no licensing, fixedfee licensing, and royalty licensing) and report the results. Section 5 extends to investigate two-part tariff licensing strategy and Section 6 concludes this paper with a discussion of the results. To simplify our exposition, all proofs are provided in the Appendix.

\section{Literature Review}

An extensive theoretical literature has been developed to explore the optimal licensing strategy. These studies can be divided into three categories as follows: (1) optimal licensing strategy considering the cost reduction innovation (e.g., [58 ], etc.), (2) vertical product innovation to improve the product quality and the optimal licensing strategy (e.g., [9$14]$, etc.), and (3) the cost reduction innovation and product innovation. For example, Li and Geng [15] studied technology licensing to the craft and product innovation of durables. They pointed out that the optimal licensing contract is determined by innovation categories and innovation scale: royalty licensing will be prioritized when it is cheaper than the reduction craft innovation or quality-improving product innovation, two-part tariff licensing will be prioritized when the scale involved is greater than that of craft innovation or vertical product innovation, and fixed-fee licensing will be prioritized when the products are average.

In this paper, we mainly study the optimal licensing strategies considering the cost reduction process innovation. Licensing strategy has received large amount of attention, for instance, Kamien and Tauman [16] studied the optimal technology licensing strategy to an external innovator using the noncooperative game theory. They pointed out that, using fixed-fee licensing, licensee firms gain lesser benefits than those with no licensing because of the fixed-fee and issuing number of licenses, whereas royalty licensing does not affect licensee firm's profits. Thus they addressed the fixed-fee licensing being significantly better than royalty licensing. Muto [17] first introduced the product substitute parameter to technology licensing considering cost reduction craft innovation and found that royalty licensing is significantly better than fixed-fee licensing when the innovation scale is comparatively small. Crama et al. [18] studied the optimal contract of $\mathrm{R} \& \mathrm{D}$ licensing. Their results reveal that the optimal licensing contract should be three-part tariff licensing, namely, payment in advance, milestone payment, and royalty because of disparities between the estimated value licensor and licensee and the limited control of the licensor over the R\&D efforts of the licensee. Mukherjee [19] figured out that as long as the Union has complete control over the bargain, licensee firms would pay lesser salaries under the royalty licensing situation than under auction or fixedfee licensing, regardless of whether the Union structure is a centralized control mode or a decentralized control mode. Royalty licensing is better than fixed-fee licensing and auction licensing for external innovators because the positive effects of low salary outweigh the negative effects of firm margin costs posed by the royalty licensing rate. 
More recently, a stream of research has emerged that explores the optimal strategy of network products. Lin and Kulatilaka [20] studied the choice of the optimal licensing strategy for duopoly markets. The results show that with the enhancement of the intensity of the network effects the optimal licensing strategy became fixed-fee licensing instead of royalty licensing. However, all network products are assumed replaceable; that is, products are completely homogeneous. However, in reality, many products have the qualities of heterogeneity. Thus, the study of optimal licensing strategy for heterogeneous products is meaningful and is a useful addition to the literature on network product technology licensing.

In addition, this paper is also related to service, contract design, and market competition. There are a growing number of research papers on these themes; we refer the reader to Choi et al. [21], Chiu et al. [22], Chan and Gao [23], Li et al. [24], $\mathrm{Xu}$ et al. [25], and so forth for reference.

The main contributions of this paper are summarized as follows. First, different with most previous research, which did not consider the network effects and substitution coefficient between different products or services in the market demand function, our model illustrates that network effects and substitution coefficient strongly influence the choice of the licensing strategy. Second, this paper indicates that the amount of R\&D investment of the competing firm, to a large extent, determines the options of the innovator's technology licensing strategies, which has not been considered in other related literature.

\section{Problem Descriptions}

3.1. Description of the Game Process. We consider a duopoly market with two competing service providers (hereafter SP for short), and both SPs can conduct new products development which experience a network effect. Suppose that one SP has developed a new technology and the other SP lagged behind temporarily, he can either develop a substitute technology by investing R\&D cost $k$ or buy the technology from the other SP. As a reminder, we call the SP who develop the new technology first the innovator SP and the SP who follows the innovator the follower SP. For the sake of simplicity, we use SP1 and SP2 to represent the innovator SP and the follower SP, respectively. SP1 can either monopolize the technology or license it to SP2. SP2 can decide whether to accept the quotation or develop a new technology by itself. To be specific, there are two cases on both SPs' competition as follows.

Case 1. SP1 chooses to monopolize the technology and SP2 can develop a new technology by itself. Both of them will then experience Cournot competition in the market. It is assumed that two products cannot be substituted and are also not compatible because of the different technological standards. However, if the market scale is not large enough, SP2 will not invest $k$ to develop a substitute innovation. In this case, SP1 becomes a monopolist and enjoys monopoly profit.

Case 2. SP1 chooses to license its technology to SP2 and proposes a licensing quotation. SP2 can choose to accept it or to refuse it. If SP2 accepts the licensing, it will experience the Cournot competition with SP1, and only one technological standard exists in the market. If SP2 refuses this licensing, it will develop new technology and two technological standards will exist in the market.

\subsection{Network Product Inverse Demand Function and Network} Intensity. The inverse demand function of formal products can be defined as $p(Q, \theta)=\theta-Q$, where $Q$ is the demand quantity of the product, $\theta$ is the market scale or the potential maximum market demand, and all SPs know the value of $\theta$. Different from the normal product, the user number can increase the additional values for the network products, and the additional values depend on the base number of users; the inverse function of the network products is $p\left(Q, q^{e}, \theta\right)=$ $\theta+v\left(q^{e}\right)-Q[20]$, where $q^{e}$ is the expectation of the network by the users and $v\left(q^{e}\right)$ is the estimated value of the network by the users and is an increasing function of $q^{e} . \theta$ is the greatest market demand when the network products do not possess the network additional values.

In the different licensing strategies, the inverse function of the network productions may be displayed in different ways. If SP1 does not license technology to SP2, for example, the no licensing strategy, SP2 makes investment $k$, then both SPs will have two incompatible standards. Thus, the users of each SP can form their own network. The products of the two SPs are incompatible so we can define the inverse function as $p_{i}=\theta+$ $v\left(q_{i}^{e}\right)-q_{i}-d q_{3-i}, i=1,2$, and $d \in[0,1]$ is the substituting rate between different products. A larger substituting rate means a tougher market competition between products; in particular, when $d=1$, the products can be substituted perfectly.

When SP1 licenses its technology to SP2 by means of fixed-fee licensing, royalty licensing, or two-part tariff licensing, both SPs compete under the same technology standard, experiencing a Cournot competition at the production stage. In this case, the users of both SPs form a larger network, resulting in higher network value. The inverse demand function is therefore given by $p=\theta+v\left(q_{1}^{e}+q_{2}^{e}\right)-q_{1}-q_{2}$.

According to Metcalfe's law, the total values of network products are proportional to the base number of the square of network users [26]. For a certain user, the value of network products can be defined as $v(q)=\beta q$ and $\beta \in[0,1)$. Note that we assume $\beta<1$ to ensure that the demand function has downward sloping or related properties. Therefore, the valid value of $\beta$ falls into $[0,1]$. This value is the network intensity that reflects the intensity of the network effects. Considering that the base numbers of the users are the same, the greater $\beta$ is, the greater the value to users brought by the network products is. However, when $\beta$ and $v(q)=0$, the network products will be degenerated as normal products.

The network effects may affect the licensing decisions of SP1 and the adoption of SP2. When SP1 does not license its technology or when both parties cannot reach an agreement on the licensing, the investment of SP2 in new products development leads to two different standards. Given this condition, only the users who buy products from the same SP will form a network, and they can simultaneously enjoy the additional network value. Thus, if these two standards are compatible, then users will not be willing to pay for 
the network products. When SPs conduct their licensing strategy, they obviously need to consider this effect.

If SP1 licenses its technology to SP2, both of them will form a large network to market products instead of two incompatible small markets. In this case, users have a greater desire to pay for the additional network value. SP1 can obtain higher price and greater benefits. Using technology licensing, SP1 can set the standard in its industry and gain licensing benefits.

\section{Three Technology Licensing Strategies}

In this section, we investigate three technology licensing strategies, namely, no licensing, fixed-fee licensing, and royalty licensing. By comparing SP1's profits of these three technology licensing strategies with product heterogeneity and network effects, we explore the optimal licensing decisions.

4.1. No Licensing Strategy. In this case, SP1 does not license technology to SP2, SP2 makes investment $k$, and then both SPs will have two incompatible standards. Thus, the users of each SP can form their own network. With the above discussion, the profit functions of both SPs are given as follows:

$$
\pi_{i}^{N}=\left[\theta+v\left(q_{i}^{e}\right)-q_{i}-q_{j}\right] q_{i}
$$

where $i, j=1,2$ and $i \neq j$ and the superscript $N$ represents the situation of no licensing.

In order to derive both SPs' optimal production decisions, we solve the first-order condition of (1) and impose a fulfilled expectation equilibrium (FEE) condition [27]. Then, we can obtain the equilibrium quantities and profits as follows:

$$
q_{i}^{*}=\frac{\theta}{2+d-\beta}, \quad \pi_{i}^{*}=\left[\frac{\theta}{2+d-\beta}\right]^{2},
$$

where $i, j=1,2$ and $i \neq j$.

Using the above function, we can obtain $\pi_{2}^{N}=\pi_{2}^{*}-k$, where $\pi_{2}^{N}$ denotes SP2's net profit from investing $k$ to develop its own standard. SP2 produces the new products only when $\pi_{2}^{N}>0$; that is, $\theta>\widehat{\theta}=(2+d-\beta) \sqrt{k}$. When $\theta \leq \widehat{\theta}$, SP2 does not produce the products or withdraw from the market. In this case, SP1 becomes the monopoly in the market and the products of SP1 are the only standard for the industry, and SP1 gains monopoly profit $\pi_{1}^{N}=\pi_{1}^{M}=[\theta /(2-\beta)]^{2}$, where the superscript $M$ represents the situation that SP1 monopolizes the market. Thus, when SP1 does not execute a license, both SPs' profits can be summarized as follows, respectively:

$$
\left(\pi_{1}^{N}, \pi_{2}^{N}\right)= \begin{cases}\left(\left[\frac{\theta}{2-\beta}\right]^{2}, 0\right), & \theta \leq \hat{\theta}, \\ \left(\left[\frac{\theta}{2+d-\beta}\right]^{2},\left[\frac{\theta}{2+d-\beta}\right]^{2}-k\right), & \theta>\hat{\theta} .\end{cases}
$$

4.2. Fixed-Fee Licensing Strategy. In this case, we consider a situation where SP1 licenses its technology to SP2 with fixedfee licensing, and SP2 pays a fixed-fee to SP1 for the technology. Both SPs compete under the same technology standard and experience a Cournot competition at the production stage. In this licensing strategy, the users of both SPs form a larger network, resulting in higher network value. The SP's profit is $\pi_{i}^{F}=\left[\theta+v\left(q_{1}^{e}+q_{2}^{e}\right)-q_{1}-q_{2}\right] q_{i}, i=1,2$, denoting SP1 and SP2, respectively. Thus, according to the conditions of FEE and the optimal production decisions of the two SPs, we can obtain the following equilibrium quantity $q_{i}^{*}=\theta /(3-2 \beta)$, $i=1,2$.

In the fixed-fee licensing strategy, the profit functions of both SPs are given by

$$
\left(\pi_{1}^{F}, \pi_{2}^{F}\right)=\left(\left[\frac{\theta}{2-3 \beta}\right]^{2}+F,\left[\frac{\theta}{2-3 \beta}\right]^{2}-F\right),
$$

where the superscript $F$ represents the fixed-fee licensing case.

At the licensing stage, SP1 decides to implement licensing and the fixed-fee is F. SP1 offers a "take-it-or-leave-it" contract to SP2. Note that when there is no difference between refusing and accepting licensing SP2 chooses to accept licensing. Thus, the optimal fixed-fee $F$ can be obtained by

$$
\begin{array}{ll}
\operatorname{Max}_{F} & \pi_{1}^{F}=\frac{\theta^{2}}{(3-2 \beta)^{2}}+F, \\
\text { s.t. } & \pi_{2}^{F}=\frac{\theta^{2}}{(3-2 \beta)^{2}}-F \geq \pi_{2}^{N} .
\end{array}
$$

According to (4), we can obtain the optimal prepaid fixedfee as follows:

$$
\begin{aligned}
F^{*} & =\frac{\theta^{2}}{(3-2 \beta)^{2}}-\pi_{2}^{N} \\
& = \begin{cases}\frac{\theta^{2}}{(3-2 \beta)^{2}}, & \theta \leq \hat{\theta}, \\
\frac{(5+d-3 \beta)(d-1+\beta) \theta^{2}}{(3-2 \beta)^{2}(2+d-\beta)^{2}}+k, & \theta>\widehat{\theta} .\end{cases}
\end{aligned}
$$

Then, we can obtain the profit of SP1 as follows:

$$
\begin{aligned}
& \pi_{1}^{F} \\
& = \begin{cases}\frac{2 \theta^{2}}{(3-2 \beta)^{2}}, & \theta \leq \hat{\theta}, \\
\frac{\left.(2+d-\beta)^{2}+(5+d-3 \beta)(d-1+\beta)\right] \theta^{2}}{(3-2 \beta)^{2}(2+d-\beta)^{2}}+k, & \theta>\widehat{\theta} .\end{cases}
\end{aligned}
$$

The profit of SP2 can be derived from (3) when SP1 charges the maximum fixed-fee. 
Theorem 1. (1) When the market is small $(\theta \leq \hat{\theta})$, if the network intensity is large $\left(\beta \geq \beta_{1}^{*}=0.293\right)$, SP1 makes more profit under fixed-fee licensing than no licensing, and then fixed-fee licensing occurs. Otherwise, for SP1, no licensing is better than fixed-fee licensing. (2) When the market scale is large $(\theta>\hat{\theta})$, if the network intensity is large $\left(\beta \geq \beta_{2}^{*}=\right.$ $1-d$ ), fixed-fee licensing will occur. Otherwise, whether fixedfee licensing is implemented for SP1 depends on the substitute rate of network products, network intensity, and ReD cost of SP2. (If the network intensity is small $\left(\beta<\beta_{2}^{*}=1-d\right)$, then we have the following. (1) When the product substitute rate is very small $\left(d<d_{1}=0.051\right)$, then regardless of R\&D cost of SP2, SP1 will choose not to implement fixed-fee licensing. (2) When the product substitute rate is very small $\left(d_{1} \leq d \leq d_{2}=0.121\right)$, then fixed-fee licensing will occur only if the network intensity is medium $\left(\beta_{3}^{*}<\beta<\beta_{4}^{*}\right)$ and $R \& D$ cost of SP2 is very high $\left(k_{2} \leq k<k_{1}\right)$. (3) When the product substitute rate is medium $\left(d_{2}<d<d_{3}=0.196\right)$, then fixed-fee licensing will occur only when the network intensity is very small $\left(\beta<\beta_{4}^{*}\right)$ and $R \& D$ cost of SP2 is very high $\left(k_{2} \leq k<k_{1}\right)$. (4) When the product substitute rate is large enough $\left(d \geq d_{3}\right)$, then as long as R\&D cost of SP2 is very high $\left(k_{2} \leq k<k_{1}\right)$, SP1 will choose to implement fixed-fee licensing.)

Theorem 1 shows that, for network products, whether SP1 licenses its technology to SP2 depends on the market scale, network intensity, product substitute rate, and SP2's R\&D cost investment to develop the new technology. When the market scale is very small $(\theta \leq \hat{\theta})$, products of SP1 can cover the entire market because of the limited market volume. In this case, if the network intensity of the products is very low $\left(\beta<\beta_{1}^{*}\right)$, then the additional network value brought by the increase in the base number of the users will not compensate for the negative effect, such as the decrease in the price as a result of overproduction. Thus, the holder of the patent should implement no licensing or monopolize the market. Unless the network is strong enough, the holder of the patent will sacrifice the monopolizing profits and choose to implement fixed-fee licensing.

When the market scale is very large $(\theta>\widehat{\theta})$ and the network intensity is strong enough $\left(\beta \geq \beta_{2}^{*}\right)$, on the one hand, the products of the holder of the patent (i.e., SP1) may not cover the whole market if the holder does not implement the licensing. SP2 can develop its own products, and if it competes with SP1, the SP1's profit will decline. On the other hand, network intensity may be strong enough. Thus, if SP1 chooses to implement licensing, then the base number of the users and network intensity will increase to improve the estimated values of users, and the profit of SP1 will improve. Thus, given this condition, SP1 will choose fixed-fee licensing. However, when network intensity is not very strong, then whether SP1 chooses to implement fixedfee licensing depends on the following aspects, among others. (1) Is the R\&D cost of SP2 high or not? (2) If SP2 successfully develops the new products, what will characterize the competition between the new products and the existing products? (3) Does a replacement exist for the network intensity and product substitute rate and the R\&D cost of SP2? These considerations lead to four conclusions (see the proof of
Theorem 1 in the Appendix). In particular, when $d=1$ and the market scale is large enough, fixed-fee licensing can definitely occur. This conclusion agrees with that made by Lin and Kulatilaka [20].

4.3. Royalty Licensing Strategy. In this case, SP1 implements technology licensing to SP2 with royalty licensing, SP2 pays a royalty rate $r$ to SP1. Both SPs follow the same technology standard and experience a Cournot competition in the market. The profit functions of both SPs are as follows:

$$
\begin{aligned}
& \pi_{1}^{R}=\left[\theta+v\left(q_{1}^{e}+q_{2}^{e}\right)-q_{1}-q_{2}\right] q_{1}+r q_{2}, \\
& \pi_{2}^{R}=\left[\theta+v\left(q_{1}^{e}+q_{2}^{e}\right)-q_{1}-q_{2}\right] q_{2}-r q_{2},
\end{aligned}
$$

where the superscript $R$ represents royalty licensing.

We can obtain the following equilibrium quantities according to the conditions of FEE and the optimal production decisions of the two SPs:

$$
q_{1}^{*}=\frac{\theta+(1-\beta) r}{3-2 \beta}, \quad q_{2}^{*}=\frac{\theta-(2-\beta) r}{3-2 \beta} .
$$
tively:

The equilibrium profits of both SPs are as follows, respec-

$$
\begin{aligned}
& \pi_{1}^{R}=\frac{\theta^{2}+(5-4 \beta) \theta r-\left(\beta^{2}-5 \beta+5\right) r^{2}}{(3-2 \beta)^{2}}, \\
& \pi_{2}^{R}=\frac{[\theta-(2-\beta) r]^{2}}{(3-2 \beta)^{2}} .
\end{aligned}
$$

And the profit function of SP2 is a decreasing function of the royalty rate because $d \pi_{2}^{R} / d r=-2(2-\beta)[\theta-(2-\beta) r] /(3-$ $2 \beta)^{2} \leq 0$. At the licensing stage, SP1 as the licensor chooses the optimal royalty rate $r_{1}^{*}$ to maximize profit, while the optimal royalty rate cannot be higher than the royalty rate $r_{2}$, which is the highest royalty rate acceptable to SP2, and we define $r^{*}=\min \left(r_{1}^{*}, r_{2}\right)$. Thus, the highest royalty rate that SP2 will accept depends on $\pi_{2}^{R}=\pi_{2}^{N L}$ because SP1 has complete power to bargain. We can obtain the following optimal royalty rate by (3) and (10). Consider

$$
r_{2}= \begin{cases}\frac{\theta}{2-\beta}, & \theta \leq \hat{\theta}, \\ \frac{\theta}{2-\beta}-\frac{3-2 \beta}{2-\beta} \sqrt{\frac{\theta^{2}}{(2+d-\beta)^{2}}-k,} & \theta>\hat{\theta} .\end{cases}
$$

According to the first-order condition, the optimal royalty rate of SP1 can be obtained from (10). We can obtain the following result:

$$
r_{1}^{*}=\frac{(5-4 \beta) \theta}{2\left(\beta^{2}-5 \beta+5\right)}
$$

We can test whether $r_{1}^{*}$ and $r_{2}^{*}$ are smaller than $\theta /(2-\beta)$, the result of which is in accordance with the upper limit 
of the royalty rate. We need to compare the values of $r_{1}^{*}$ and $r_{2}$ to obtain the optimal royalty rate $r^{*}=\min \left(r_{1}^{*}, r_{2}\right)$. The following theorem yields the optimal royalty rate and its conditions.

Theorem 2. In royalty licensing mode, the optimal royalty rate of SP1 depends on the critical value $\tilde{\theta}$ of the market scale. When $\theta \leq \widetilde{\theta}$, the optimal royalty rate is $r^{*}=r_{1}^{*}$; when $\theta>\widetilde{\theta}$, the optimal royalty rate is $r^{*}=r_{2}$, where

$$
\tilde{\theta}=\frac{2(2+d-\beta)\left(5-5 \beta+\beta^{2}\right) \sqrt{k}}{\sqrt{\left[10+(d-8) \beta+\beta^{2}\right]\left[10-(12+d) \beta+3 \beta^{2}\right]}}
$$

Thus, we can obtain both SPs' profits at the licensing stage as follows:

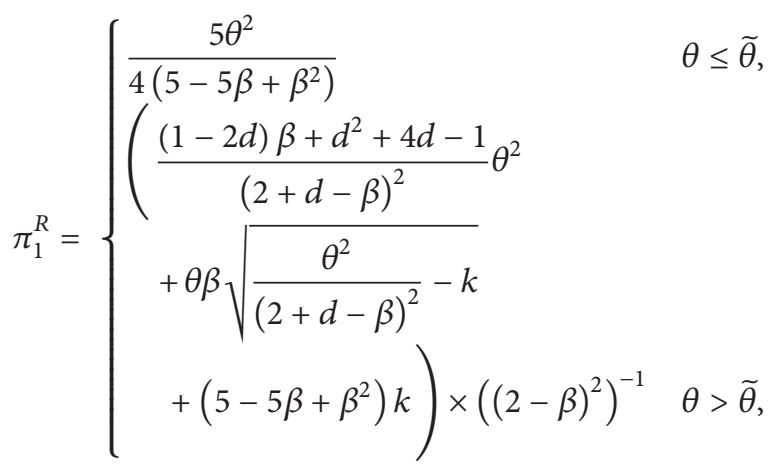

$$
\pi_{2}^{R}= \begin{cases}\frac{\theta^{2} \beta^{2}}{4\left(5-5 \beta+\beta^{2}\right)^{2}} & \theta \leq \tilde{\theta}, \\ \frac{\theta^{2}}{(2+d-\beta)^{2}}-k & \theta>\widetilde{\theta}\end{cases}
$$

Under royalty licensing, SP2 is always prepared to accept licensing because of $\Delta \pi_{2}=\pi_{2}^{R}-\pi_{2}^{N} \geq 0$. The question is whether or not SP1 will implement licensing. The following results provide the conditions under which SP1 chooses to implement licensing.

Theorem 3. When the market scale is comparatively small $(\theta \leq \widetilde{\theta})$, then for any network intensity $\beta \in[0,1)$, royalty licensing will always occur. When the market scale is comparatively large $(\theta>\widetilde{\theta})$, then the occurrence of royalty licensing depends on the product substitution rate, network intensity, and the R\&D cost of SP2.

Theorem 3 shows that the increase in the market scale aids the increase in the profit of SP1. When the market scale is large enough, the product substitute rate, network intensity, and $R \& D$ cost of the potential licensee determine the final decision of SP1. When the market scale is very small $(\theta \leq$ $\widehat{\theta})$ and when the network product is regarded as a normal product $(\beta=0)$, SP1 will have no profits when it implements royalty licensing because the market is not large enough for more firms to enter. If more firms participate in the competition, the overall profits will be close to zero. Thus, SP1, as the one with the monopoly potential, should not implement the licensing. In terms of network products $(\beta>0)$, stiffer competition among firms will decrease the industry profits, but users give higher comments on the increase in network scale, indicating higher additional network value and more industry profits. Given this condition, the enlargement of the market scale resulting from the positive effect (i.e., network effects) and the licensing benefits resulting from licensing outweigh the negative effect, which is the increase in the competition resulting from licensing.

Considering the enlargement of the market scale $(\widehat{\theta}<\theta \leq$ $\widetilde{\theta})$, the network effect will decrease because if the network products are regarded as normal products, SP1 will also choose to implement royalty licensing. Thus, firms need to consider the values of the positive effect caused by licensing and the negative effect caused by competition. However, users of different companies after licensing will form a larger network and produce greater additional network value, thus leading to the positive effect caused by licensing that is greater than the negative effect caused by competition. The potential licensee can develop its own products and thus, to some extent, cause the opportunity cost to be higher without licensing. After overall consideration, we find that the positive effect is higher. Thus, royalty licensing is better to be implemented when the market scale is larger.

Once the market scale is large enough $(\theta>\widetilde{\theta})$, on the one hand, SP1 will not be concerned about whether the market is large enough for more companies but about whether the market for network products can compensate for the losses after licensing under the larger and the same standard network product market. The larger the network intensity $\beta$, the stronger the motivation of SP1 to implement royalty licensing. On the other hand, the stiffness of the competition between SP1 and SP2 under no licensing and when the standard is not the same should be considered. The larger is the product substitute rate $d$ (which reflects the stiffness of the competition between the products), the stronger the motivation to implement licensing for SP1. SP1 will also consider the motivation that SP2 accepts the licensing. The higher the R\&D cost of SP2, the stronger the motivation to accept the licensing. The lower the R\&D cost of SP2, the stronger its motivation to develop new products. Under royalty licensing, the lower the R\&D cost of SP2, the stronger the motivation of SP1 to implement the licensing.

\section{Optimal Licensing Strategies}

In this section, we discuss the optimal licensing strategy by comparing SP1's profits under no licensing, fixed-fee licensing, and royalty licensing strategy. This helps us better understand the conditions under which SPs choose to implement and accept the technology licensing.

When the market scale is small $(\theta \leq \widetilde{\theta})$, fixed-fee licensing strategy can be used only for SP1 when the network intensity is large enough $\left(\beta>\beta_{1}^{*}=0.293\right)$, and royalty licensing is not influenced by network intensity $\beta$. Then, using the comparison of profits between the two licensing strategies, we 
can obtain $\pi_{1}^{F}=\pi_{1}^{R}$ when $\beta_{8}^{*}=(5-\sqrt{10}) / 6 \approx 0.306, \pi_{1}^{F}<\pi_{1}^{R}$ when $\beta<\beta_{8}^{*}$, and $\pi_{1}^{F}>\pi_{1}^{R}$ when $\beta>\beta_{8}^{*}$.

When the market scale is medium $(\widehat{\theta}<\theta \leq \widetilde{\theta})$, fixedfee licensing can be used under four conditions, and royalty licensing always occurs. Thus, we need to compare SP1's profits given fixed-fee licensing and royalty licensing under the four kinds of conditions. After the comparison, we can determine that R\&D cost of SP2 is $k_{5}=k_{2}+\left(\left((\beta-5 d)^{2}+\right.\right.$ $\left.20 d(1-d)) / 4(2+d-\beta)^{2}\left(5-5 \beta+\beta^{2}\right)\right) \theta^{2}$, and we obtain $\pi_{1}^{F}=\pi_{1}^{R}$ when $k_{5}<k<k_{1}$ and $\pi_{1}^{F}>\pi_{1}^{R}$ when $k_{2}<k<k_{5}$. We can also obtain $k_{5}=k_{1}$ when $\beta_{9}^{*} \approx 0.319, k_{5}>k_{1}$ when $\beta<\beta_{9}^{*}$, and $k_{5}<k_{1}$ when $\beta>\beta_{9}^{*}$. We can determine the following: (1) when $\beta<\beta_{9}^{*}$, for any $k<k_{1}$, we obtain $\pi_{1}^{F}<\pi_{1}^{R}$; (2) when $\beta>\beta_{9}^{*}$, if $k_{2}<k<k_{5}$, we obtain $\pi_{1}^{F}<\pi_{1}^{R}$, and if $k_{5}<k<k_{1}$, we obtain $\pi_{1}^{F}>\pi_{1}^{R}$.

However, when the market scale is large enough $(\theta>\tilde{\theta})$, the licensing strategy becomes very complicated. Fixed-fee licensing can occur under four conditions, and royalty licensing can occur under seven conditions. Fifteen conditions can ensure the occurrence of both licensing methods. Thus, the optimal strategy can be obtained only by comparing the 15 conditions. However, the profit functions of the fifteen conditions are the same considering the same licensing strategy. For convenience, we first compare the profits and then input the limited conditions to test them. After comparing $\pi_{1}^{F}$ under fixed-fee licensing and $\pi_{1}^{R}$ under royalty licensing for SP1, we obtain $\pi_{1}^{R}=\pi_{1}^{F}$ when $\mathrm{R} \& \mathrm{D}$ cost of SP2 is

$$
k_{6}=\frac{H_{4}}{(2+d-\beta)^{2}(3-2 \beta)^{2}(1-\beta)} \theta^{2},
$$

where

$$
\begin{aligned}
H_{4}= & {\left[-2 \beta^{2}+2(2-3 d) \beta+2 d^{2}+8 d-1\right](2-\beta)^{2} } \\
& -\left[(1-2 d) \beta+d^{2}+4 d-1\right](3-2 \beta)^{2} .
\end{aligned}
$$

When $k_{6}<k<k_{1}, \pi_{1}^{F}<\pi_{1}^{R}$; when $k<k_{6}, \pi_{1}^{F}>\pi_{1}^{R}$. Finally, we compare $k_{6}$ with the 15 critical values, and then we obtain the eight conditions that enable the simultaneous occurrence of both licensing strategies. All the conclusions are summarized in Table 1.

Analyzing all the above results, we can obtain Proposition 4.

Proposition 4. (1) When the market scale is small $(\theta \leq \widehat{\theta})$, if $\beta_{1}^{*} \leq \beta<\beta_{8}^{*}$, royalty licensing is better than fixed-fee licensing and if $\beta>\beta_{8}^{*}$, fixed-fee licensing is better than royalty licensing.

(2) When the market scale is medium $(\widehat{\theta}<\theta \leq \widetilde{\theta})$ or large enough $(\theta>\widetilde{\theta})$, the degree of the differentiation of the products, the intensity of the network effects, and the R\&D cost of SP2 play a key role in determining the optimal licensing strategy of SP1.

The result of Proposition 4 has the following implications for SPs' licensing strategies. Firstly, when the market scale is small $(\theta \leq \hat{\theta})$, for any positive network intensity, implementing technology licensing is better for SP1. This implication also indicates that obtaining monopoly profits is not the optimal choice.

Secondly, when the market scale is medium $(\hat{\theta}<\theta \leq \widetilde{\theta})$, developing new technologies and challenging the monopoly position of SP1 are possible for SP2. When SP2 chooses its own technology and competes with SP1 in the market, the incomplete substitute of the products reflects the intensity of the competition between the companies. Moreover, the prediction of fierce competition between two SPs and the consideration of SP2's profitability will influence SP1's attitude toward technology licensing and the SP's approach to its implementation. Thus, predictably, whether SP1 will choose to implement the technology licensing depends on the product substitution rate $d$ and SP2's R\&D cost $k$, except for the network intensity $\beta$.

Finally, when the market scale is large enough $(\theta>$ $\widetilde{\theta})$, there is no certainty that the two strategies (i.e., fixedfee licensing and royalty licensing) will always be better than no licensing. This situation shows that except for the aforementioned key parameters (i.e., network intensity $\beta$, product substitution rate $d$, and SP2's R\&D cost $k$ ) technology licensing is also influenced by market scale $\theta$. Based on the critical values of the various kinds of functions of SP2's R\&D cost, the market scale $\theta$ exerts influence on the essence. Thus, implementing technology licensing is not always optimal for SP1. By determining that two licensing methods can occur at the same time, we can reach the conclusion of $\theta>\widetilde{\theta}$ in Proposition 4.

\section{Two-Part Tariff Licensing}

In this section, we consider a two-part tariff licensing strategy, where SP1 charges a prepaid fixed-fee and a royalty per unit of SP2's output. In normal product market, two-part tariff licensing mechanism, with its complete and flawless information, is superior to both fixed-fee licensing and royalty licensing mechanisms. Is it still the optimal mechanism or strategy for SP1 in the network product market? To answer this question, we propose a model where SP1 licenses its technology to SP2 by means of two-part tariff licensing in the network product market.

Under two-part tariff licensing, the profit functions of both SPs are as follows:

$$
\begin{aligned}
& \pi_{1}^{F R}=\left[\theta+v\left(q_{1}^{e}+q_{2}^{e}\right)-q_{1}-q_{2}\right] q_{1}+r q_{2}+F \\
& \pi_{2}^{F R}=\left[\theta+v\left(q_{1}^{e}+q_{2}^{e}\right)-q_{1}-q_{2}\right] q_{2}-r q_{2}-F
\end{aligned}
$$

where the superscript FR represents two-part tariff licensing.

Solving the above problems yields the following profits:

$$
\begin{aligned}
& \pi_{1}^{F R}=\frac{\theta^{2}+(5-4 \beta) \theta r-\left(\beta^{2}-5 \beta+5\right) r^{2}}{(3-2 \beta)^{2}}+F, \\
& \pi_{2}^{F R}=\frac{[\theta-(2-\beta) r]^{2}}{(3-2 \beta)^{2}}-F .
\end{aligned}
$$


TABLE 1: Conditions that enable the simultaneous occurrence of both licensing strategies.

\begin{tabular}{lcc}
\hline Product substitution rate and network intensity condition & R\&D cost condition & Profits relationship \\
\hline$d>d_{4}=0.414$ and $\beta>\beta_{5}^{*}$ & $k_{6}<k<k_{1}$ & $\pi_{1}^{F}<\pi_{1}^{R}$ \\
$d>d_{4}=0.414$ and $\beta_{2}^{*} \leq \beta<\beta_{5}^{*}$ & $k>k_{6}$ & $\pi_{1}^{F}>\pi_{1}^{R}$ \\
$d \leq d_{4}=0.414$ and $\beta>\beta_{2}^{*}$ & $k \leq \min \left\{k_{6}, k_{4}\right\}=k_{4}$ & $\pi_{1}^{F}>\pi_{1}^{R}$ \\
$0.1964=d_{3} \leq d \leq d_{5}=0.314$ and $\beta_{7}^{*} \leq \beta<\beta_{2}^{*}$ & $k \leq k_{4}$ & $\pi_{1}^{F}>\pi_{1}^{R}$ \\
$d>d_{5}$ and $\beta<\beta_{2}^{*}$ & $k_{2} \leq k<k_{1}$ & $\pi_{1}^{F}<\pi_{1}^{R}$ \\
$d_{3} \leq d \leq d_{5}$ and $\beta<\beta_{7}^{*}$ & $k_{2} \leq k<k_{3}$ & $\pi_{1}^{F}<\pi_{1}^{R}$ \\
$d_{2}<d<d_{3}$ and $\beta<\beta_{4}^{*}$ & $k_{2} \leq k<k_{3}$ & $\pi_{1}^{F}<\pi_{1}^{R}$ \\
$d_{1} \leq d \leq d_{2}$ and $\beta_{3}^{*}<\beta<\beta_{4}^{*}$ & $k_{2} \leq k<k_{3}$ & $\pi_{1}^{F}<\pi_{1}^{R}$ \\
$d>d_{4}=0.414$ and $\beta>\beta_{5}^{*}$ & $k_{2} \leq k<k_{3}$ & $\pi_{1}^{F}<\pi_{1}^{R}$ \\
$d>d_{4}=0.414$ and $\beta_{2}^{*} \leq \beta<\beta_{5}^{*}$ & $k_{6}<k<k_{1}$ & $\pi_{1}^{F}<\pi_{1}^{R}$ \\
$d \leq d_{4}=0.414$ and $\beta>\beta_{2}^{*}$ & $k>k_{6}$ & $\pi_{1}^{F}>\pi_{1}^{R}$ \\
$0.1964=d_{3} \leq d \leq d_{5}=0.314$ and $\beta_{7}^{*} \leq \beta<\beta_{2}^{*}$ & $\pi_{1}^{F}>\pi_{1}^{R}$ \\
$d>d_{5}$ and $\beta<\beta_{2}^{*}$ & $\pi_{1}^{F}>\pi_{1}^{R}$ \\
$d_{3} \leq d \leq d_{5}$ and $\beta<\beta_{7}^{*}$ & $\left.k k_{6}, k_{4}\right\}=k_{4}$ & $\pi_{1}^{F}<\pi_{1}^{R}$ \\
$d_{2}<d<d_{3}$ and $\beta<\beta_{4}^{*}$ & $k_{2} \leq k<k_{1}$ & $\pi_{1}^{F}<\pi_{1}^{R}$ \\
$d_{1} \leq d \leq d_{2}$ and $\beta_{3}^{*}<\beta<\beta_{4}^{*}$ & $k_{2} \leq k<k_{3}$ & $\pi_{1}^{F}<\pi_{1}^{R}$ \\
& $k_{2} \leq k<k_{3}$ & $\pi_{1}^{F}<\pi_{1}^{R}$
\end{tabular}

The optimal fixed-fee and royalty rate should maximize SP1's profit as long as SP2 agrees to license. In other words, the optimal rate fixed-fee $F^{*}$ and royalty $r *$ should solve

$$
\max _{F, r} \pi_{1}^{F R}=\frac{\theta^{2}+(5-4 \beta) \theta r-\left(\beta^{2}-5 \beta+5\right) r^{2}}{(3-2 \beta)^{2}}+F
$$

such that

$$
\pi_{2}^{F R}=\frac{[\theta-(2-\beta) r]^{2}}{(3-2 \beta)^{2}}-F \geq \pi_{2}^{N}
$$

When the market scale is small $(\theta \leq \hat{\theta})$, solving the above problem yields SP1's optimal fixed-fee:

$$
F_{1}^{*}=\pi_{2}^{F R}-\pi_{2}^{N}=\frac{[\theta-(2-\beta) r]^{2}}{(3-2 \beta)^{2}}
$$
rate

Integrating (23) into (21), we obtain the optimal royalty

$$
r_{3}^{*}= \begin{cases}\frac{(1-2 \beta) \theta}{2(1-\beta)}, & \beta<\beta_{10}^{*}=0.5 \\ 0, & \beta \geq 0.5 .\end{cases}
$$

Thus, we have the optimal fixed-fee

$$
F_{1}^{*}= \begin{cases}\frac{\theta^{2} \beta^{2}}{4(1-\beta)^{2}} & \beta<0.5, \\ \frac{\theta^{2}}{(3-2 \beta)^{2}} & \beta \geq 0.5 .\end{cases}
$$

In the following, we will compare SP1's profits under different licensing strategies. When $\beta \geq 0.5$, the optimal royalty rate is 0 ; that is, two-part tariff licensing degrades to fixed-fee licensing. Then, we obtain $\pi_{1}^{F R}=\pi_{1}^{F}=2 \theta^{2} /(3-2 \beta)^{2}$. According to the conclusion in Case (1) of Proposition 4, we have $\pi_{1}^{F}>\pi_{1}^{R}$. Thus, $\pi_{1}^{F R}>\pi_{1}^{R}$. When $\beta<0.5$, we compare $\pi_{1}^{F R}$ with $\pi_{1}^{R}$. Substituting (24) and (25) into (20) and combining (6) and (14), we have

$$
\begin{aligned}
& \pi_{1}^{F R}-\pi_{1}^{F}=\frac{(1-2 \beta) \theta}{2(3-2 \beta)^{2}} r_{3}^{*}>0, \\
& \pi_{1}^{F R}-\pi_{1}^{R}=\frac{H_{5}}{4(3-2 \beta)^{2}\left(5-5 \beta+\beta^{2}\right)(1-\beta)},
\end{aligned}
$$

where $H_{5}=\left(-5+20 \beta-12 \beta^{2}\right)(1-\beta)+(1-2 \beta)^{2}\left(5-5 \beta+\beta^{2}\right)$. As shown in Figure $1, H_{5}>0$. Then, we have $\pi_{1}^{F R}-\pi_{1}^{R}>0$.

Based on the above problem, we have the following proposition.

Proposition 5. In a small market $(\theta \leq \widehat{\theta})$, if all three licensing strategies are valid, then two-part tariff licensing degrades to fixed-fee licensing when $\beta \geq 0.5$. For SP1, the optimal licensing strategy is fixed-fee licensing. When $\beta<0.5$, the optimal licensing strategy is two-part tariff licensing.

When the market demand is in the medium range $(\widehat{\theta}<$ $\theta \leq \widetilde{\theta}$ ), it is possible for SP2 to develop new technology if it does not implement licensing. Based on (3), we can derive that

$$
F_{2}^{*}=\pi_{2}^{F R}-\pi_{2}^{N}=\frac{[\theta-(2-\beta) r]^{2}}{(3-2 \beta)^{2}}-\frac{\theta^{2}}{(2+d-\beta)^{2}}+k .
$$




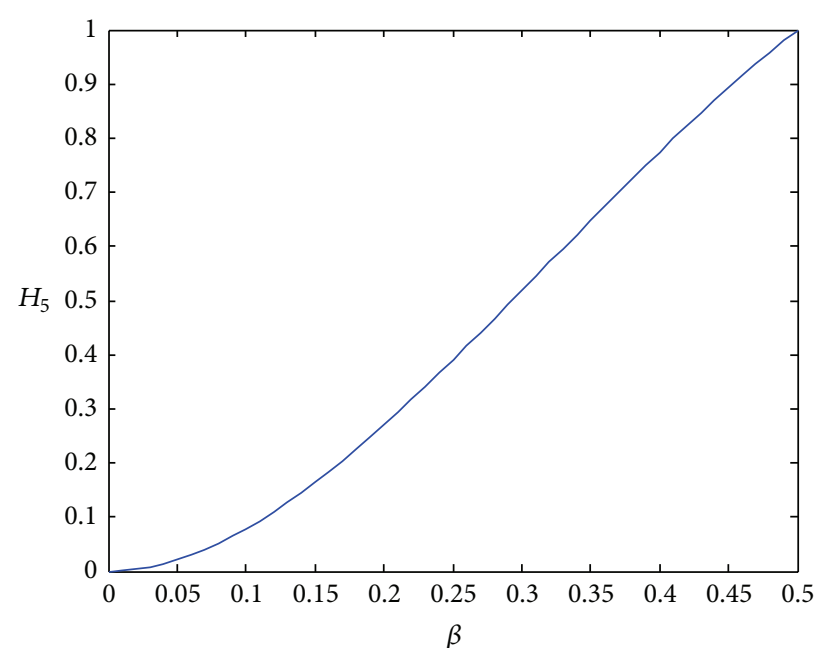

FIgure 1: Changing of $H_{5}$ on $\beta \in[0,0.5)$.

Based on (19), the optimal royalty rate is the same as (24). Thus, the optimal fixed-fee is

$$
F_{2}^{*}= \begin{cases}\frac{\theta^{2} \beta^{2}}{4(1-\beta)^{2}}-\frac{\theta^{2}}{(2+d-\beta)^{2}}+k & \beta<0.5, \\ \frac{(5+d-3 \beta)(\beta-1+d) \theta^{2}}{(3-2 \beta)^{2}(2+d-\beta)^{2}}+k & \beta \geq 0.5 .\end{cases}
$$

The same methods are applied in the following parts as we compare $\pi_{1}^{F R}$ with $\pi_{1}^{R}$ and $\pi_{1}^{F}$ under different rates of network intensity. When $\beta \geq 0.5$, the royalty rate is 0 under twopart tariff licensing, it simplifies to be a fixed-fee licensing. Thus, $\pi_{1}^{F R}=\pi_{1}^{F}$. Therefore, the profit comparison under twopart tariff licensing and royalty licensing essentially becomes the profit comparison under fixed-fee licensing and royalty licensing. According to the case of $\widehat{\theta}<\theta \leq \widetilde{\theta}$ in Proposition 4 and in conjunction with the critical value under network intensity, when $\beta \geq \max \left(\beta_{2}^{*}, \beta_{10}^{*}\right)$, if $k_{5} \leq k<k_{1}$, we have $\pi_{1}^{F R}=\pi_{1}^{F}>\pi_{1}^{R}$; if $k<k_{5}$, then $\pi_{1}^{F R}=\pi_{1}^{F}<\pi_{1}^{R}$.

Next, we explore SPl's optimal licensing strategy when $\beta<0.5$. Based on (24) and (28) and the profit of SP1 under fixed-fee licensing, we have

$$
\begin{aligned}
& \pi_{1}^{F R}-\pi_{1}^{F}=\frac{\left[(1-2 \beta) \theta-(1-\beta) r_{3}^{*}\right] r_{3}^{*}}{(3-2 \beta)^{2}}>0, \\
& \pi_{1}^{F R}-\pi_{1}^{R}=k-k_{7}=k-\left[k_{1}-\frac{\theta^{2} \beta^{2}}{4(1-\beta)\left(5-5 \beta+\beta^{2}\right)}\right],
\end{aligned}
$$

where

$$
\begin{gathered}
k_{7}=-\frac{H_{6}}{4(2+d-\beta)^{2}\left(5-5 \beta+\beta^{2}\right)(1-\beta)} \theta^{2}, \\
H_{6}=\beta^{2}(2+d-\beta)^{2}-4(1-\beta)\left(5-5 \beta+\beta^{2}\right) .
\end{gathered}
$$

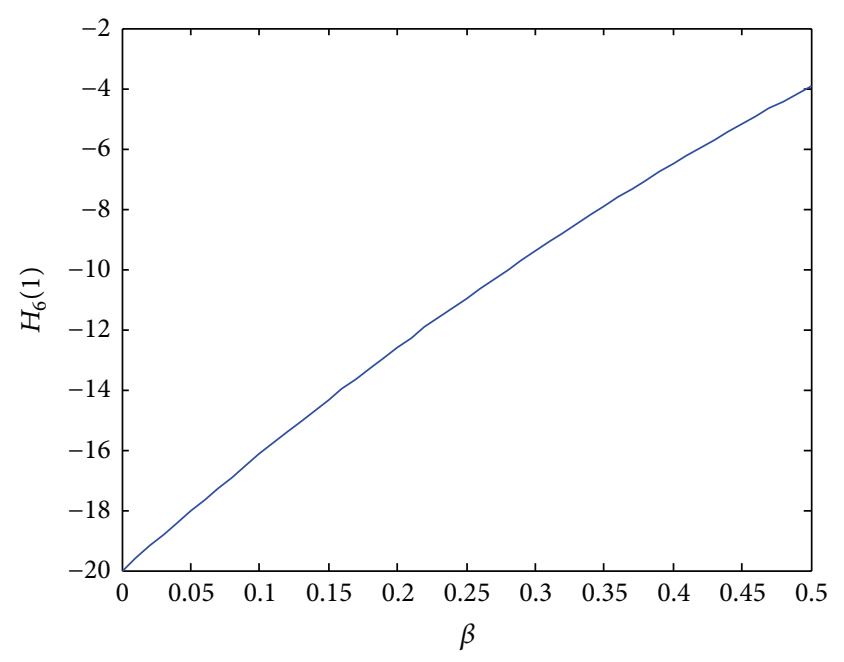

FIGURE 2: Changing of $H_{6}(d)$ regarding $\beta$ when $d=1$.

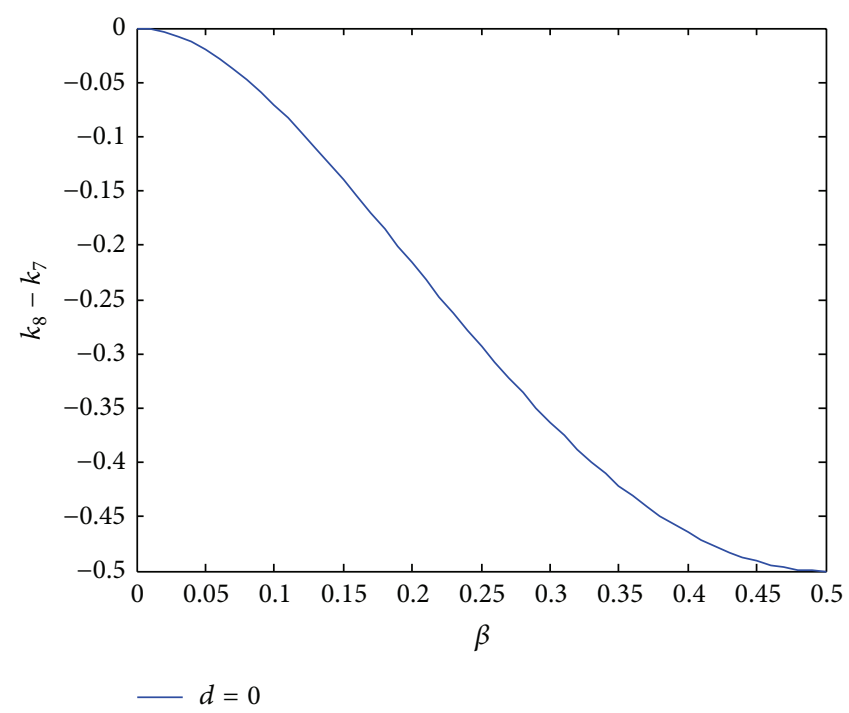

FIgURE 3: Changing of the critical value $k_{8}-k_{7}$ regarding $\beta$ when $d=0$.

Clearly, $H_{6}(d)$ is the increasing function of $d \in[0,1]$. Figure 2 indicates that $H_{6}(1)<0$, so we can easily obtain $0<k_{7} \leq k_{1}$.

The result shows that $\pi_{1}^{F R}>\pi_{1}^{R}$, when $k_{7}<k<k_{1}$ and $\pi_{1}^{F R}<\pi_{1}^{R}$, when $k<k_{7}$. In the medium market scale, the validity of the two-part system depends on the comparison between $\pi_{1}^{F R}$ and $\pi_{1}^{N}$. As $\pi_{1}^{F R}-\pi_{1}^{N}=k-k_{8}$, only when $k>$ $k_{8}$ does two-part tariff licensing become valid, where $k_{8}=$ $\left(\left(8(5+d-3 \beta)(1-d-\beta)(1-\beta)-(1-2 \beta)^{2}(2+d-\beta)^{2}\right) / 4(3-\right.$ $\left.2 \beta)^{2}(2+d-\beta)^{2}(1-\beta)\right) \theta^{2}$. As Figures 3 and 4 show that $k_{8}-k_{7}<$ 0 . Therefore, the aforementioned conclusions can guarantee the validity of the two-part tariff licensing strategy.

Based on the conclusions under different rates of network intensity, when the market scale is in the medium range $(\widehat{\theta}<$ $\theta \leq \tilde{\theta})$, we obtain the following two propositions. 


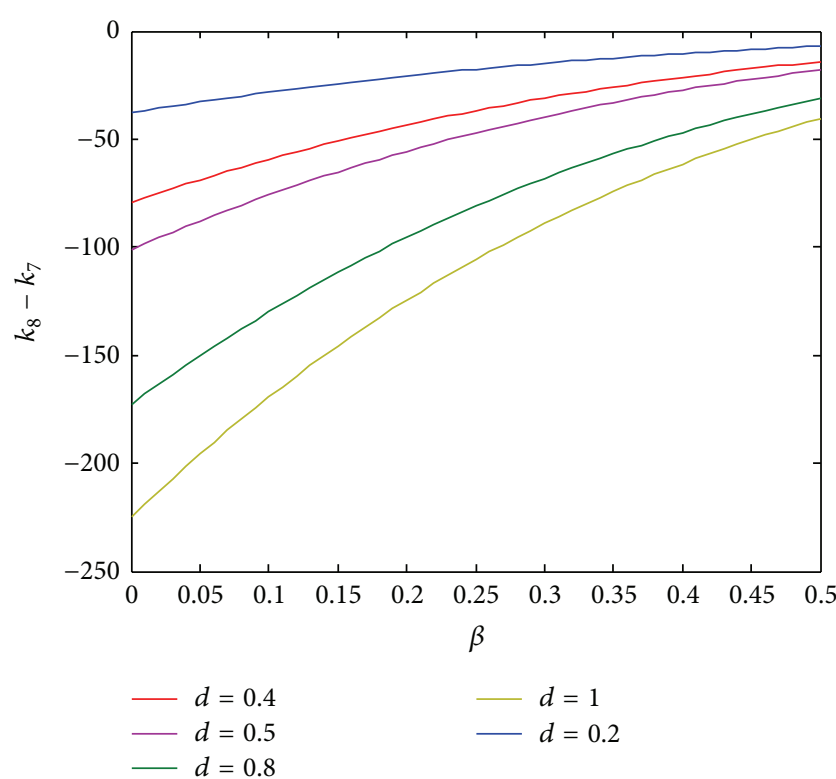

FIGURE 4: Changing of the critical value $k_{8}-k_{7}$ under different $d$ regarding $\beta$.

Proposition 6. When $\beta \geq 0.5$, two-part tariff licensing degrades to fixed-fee licensing. As for SP1, three licensing strategies will not occur only when the network intensity is relatively high (e.g., $\beta \geq \max \left\{\beta_{2}^{*}, \beta_{10}^{*}\right\}$ ). If SP2's R\&D cost is relatively high (e.g., $k_{5} \leq k<k_{1}$ ), then fixed-fee licensing will be the optimal strategy. If SP2's R\&D cost is relatively low (e.g., $\left.k<k_{5}\right)$, then royalty licensing will be the optimal strategy.

Proposition 7. When $\beta<0.5$, as far as SP1 is concerned, its optimal licensing strategy relies on the R\&D cost of SP2. If SP2's R\&D cost is relatively high (e.g., $k_{5} \leq k<k_{1}$ ), then two-part tariff licensing will be the optimal strategy. If SP2's R\&D cost is relatively low (e.g., $k<k_{7}$ ), then royalty licensing will be the optimal strategy.

However, when the market scale is relatively large $(\theta>$ $\widetilde{\theta})$, the optimal fixed-fee and royalty rate are the same as those under the condition of medium market scale. When network intensity is relatively high (e.g., $\beta \geq 0.5$ ), the optimal royalty rate is zero. In this case, two-part tariff licensing is equal to fixed-fee licensing. By comparing the profits between fixed-fee licensing and royalty licensing, we can determine the optimal licensing strategy. The comparison of fixed-fee licensing and royalty licensing is provided in the case of $\theta>\widetilde{\theta}$ of Proposition 4. Therefore, we only have to consider what will happen under low network intensity (e.g., $\beta<0.5$ ). Similar to the situation under a medium market scale, twopart tariff licensing is always superior to fixed-fee licensing. Then, we only have to compare the value of two-part tariff licensing with that of royalty licensing.

$$
\text { As } \pi_{1}^{F R}-\pi_{1}^{R}=(1-\beta)\left[\sqrt{\theta^{2} /(2+d-\beta)^{2}-k}-\beta \theta / 2(1-\right.
$$
$\beta)]^{2} /(2-\beta)^{2}>0$, when network intensity is relatively low (e.g., $\beta<0.5$ ), two-part tariff licensing is always superior to fixed-fee licensing and royalty licensing. Subsequently, we have Proposition 7 at a higher market scale.

Proposition 8. When the market scale is high $(\theta>\widetilde{\theta})$. If all the three licensing strategies are valid, then (1) when $\beta \geq 0.5$, twopart tariff licensing strategy is the same as fixed-fee licensing strategy. In this case, the optimal licensing strategy is given by Proposition 4. (2) When $\beta<0.5$, as for SP1, the two-part tariff licensing strategy is always superior to fixed-fee licensing and royalty licensing strategy.

Propositions 5 to 8 suggest that, under two-part tariff licensing, royalty licensing and fixed-fee licensing are possible as long as the network intensity is not extremely high (e.g., $\beta<0.5$ ), and SP2's R\&D cost is high enough (e.g., $\left.k_{7} \leq k<k_{1}\right)$. Then, the optimal strategy of SP1 is invariably two-part tariff licensing, which contains the merits of both fixed-fee licensing and royalty licensing.

However, because of the particularity of the network product market and the existence of a market scale, product substitution level, R\&D cost of SP2, and especially network effects, two-part tariff licensing does not always turn out to be the optimal strategy. This situation is especially true when there are extreme parameters in the R\&D cost of SP2 and network intensity. Consider the following examples. (1) When the market scale is relatively small and the network intensity is relatively high, the network effect is better than the supply side effect. Fixed-fee licensing develops its advantage under stronger network effects and a smaller market scale. Thus, fixed-fee licensing will be better than royalty licensing and two-part tariff licensing in the middle position. (2) When the market scale is medium and the network intensity is extremely high because of the increasingly larger market scale, fixed-fee licensing becomes less influential on the market. Therefore, we have to consider SP2's R\&D cost when the network intensity reaches a certain critical level. As stated in Proposition 4, high R\&D cost is beneficial to the implementation of fixed-fee licensing and low R\&D cost forces SP1 to implement royalty licensing to keep its leading position in terms of output. (3) When the market scale is large and the network intensity is relatively high, the influence of fixed-fee licensing and royalty licensing on the market is remarkably weakened, and royalty licensing has an even weaker impact. Then, SP1 has to consider SP2's R\&D cost and also anticipate its product substitution level.

\section{Conclusions}

In this paper, we have examined the technology licensing problems of network product in a duopolistic SP competition market. We also compared the SPs profits in the four licensing strategies: no licensing, fixed-fee licensing, royalty licensing, and two-part tariff licensing strategy. We have shown that the licensing strategy choices depend on the combination of the market scale, the network product substitution rate, the network effect, and SP's R\&D cost.

When the network products can be completely substituted, two-part tariff licensing is always the optimal strategy for the incumbent SP in the normal and network product 
markets. This finding is in accordance with the conclusion made by Lin and Kulatilaka [20]. However, when the network products cannot be completely substituted, two-part tariff licensing is not always optimal. This condition shows that no ideal strategy constantly works and that the conclusion that two-part tariff licensing is the optimal choice in the normal product market or in the network product market, in which all products can be substituted completely, is only a special case. Thus, if we want to know which strategy is optimal, we have to consider the combinations of all the aforementioned parameters, the details of which are in the conclusions of Theorems 1 to 3 and Propositions 4 to 8 .

We now discuss some limitations in this paper and possible avenues for future research. First, our analysis focused on the assumption of information symmetry (i.e., all parameters are common knowledge). In reality, SP2's R\&D cost is often private information. If SP1's decision is based upon the asymmetric information, then our results may not hold. Second, we assume that SP1 has a perfect capacity for bargaining, he can give SP2 an "accept-it-or-refuse-it" offer. Our results might make a big difference if we assume SP1 could not have the perfect capacity for bargaining. Third, we consider there are only two competing SPs in the industry, neglecting the superior supplier of the supply chain. When there exist upstream enterprises, SP1's licensing strategy and the outputs of the two SPs might change a lot. All of these limitations leave us a fertile area for future research.

\section{Appendix}

\section{Relative Parameters in a Duopoly Competition}

Consider

$$
\begin{aligned}
& k_{1}=\frac{\theta^{2}}{(2+d-\beta)^{2}}, \quad k_{2}=\frac{2(5+d-3 \beta)(1-d-\beta) \theta^{2}}{(3-2 \beta)^{2}(2+d-\beta)^{2}}, \\
& k_{3}=\frac{-C_{4}+\sqrt{\Delta_{1}}}{2 C_{3}}, \\
& k_{4}=\frac{\beta^{2}-(5-2 d) \beta-d^{2}-4 d+5}{\left(5-5 \beta+\beta^{2}\right)(2+d-\beta)^{2}} \theta^{2}, \\
& k_{5}=k_{2}+\frac{\beta^{2}-10 d \beta+20 d+5 d^{2}}{4(2+d-\beta)^{2}\left(5-5 \beta+\beta^{2}\right)} \theta^{2} \text {, } \\
& k_{6}=\frac{H_{4}}{(2+d-\beta)^{2}(3-2 \beta)^{2}(1-\beta)} \theta^{2} \text {, } \\
& k_{7}=-\frac{H_{6}}{4(2+d-\beta)^{2}\left(5-5 \beta+\beta^{2}\right)(1-\beta)} \theta^{2}, \\
& k_{8}=(8(5+d-3 \beta)(1-d-\beta)(1-\beta) \\
& -(1-2 \beta)^{2}(2+d-\beta)^{2} \\
& \left.\times\left(4(3-2 \beta)^{2}(2+d-\beta)^{2}(1-\beta)\right)^{-1}\right) \theta^{2},
\end{aligned}
$$

$$
\begin{gathered}
\beta_{1}^{*}=0.293, \quad \beta_{2}^{*}=1-d, \\
\beta_{3}^{*}=\frac{(1-d)-\sqrt{17 d^{2}+62 d-7}}{4}, \\
\beta_{4}^{*}=\frac{(1-d)+\sqrt{17 d^{2}+62 d-7}}{4}, \\
\beta_{5}^{*}=\frac{5-2 d-\sqrt{5-4 d+8 d^{2}}}{2}, \\
\beta_{6}^{*}=\frac{5-2 d+\sqrt{5-4 d+8 d^{2}}}{2}, \\
\beta_{7}^{*}=\frac{-2 d^{3}-16 d^{2}+\sqrt{\Delta_{2}}}{2\left(4 d^{2}-40 d+50\right)}, \\
\beta_{8}^{*}=0.306, \quad \beta_{9}^{*} \approx 0.319, \quad \beta_{10}^{*}=0.5, \\
d_{1}=0.051, \quad d_{2}=0.121, \quad d_{3}=0.196, \\
d_{4}=0.414, \quad d_{5}=0.314 .
\end{gathered}
$$

Proof of Theorem 1. If fixed-fee licensing is to be implemented, then there has to be $\Delta \pi_{1}=\pi_{1}^{F}-\pi_{1}^{N L} \geq 0, \Delta \pi_{2}=$ $\pi_{2}^{F}-\pi_{2}^{N L} \geq 0$. As the SP2's profits remain unchanged before and after licensing (because SP1 is completely capable of bargaining), it is automatically qualified to accept licensing. Accordingly, the implementation of fixed-fee licensing depends on the condition $\Delta \pi_{1}=\pi_{1}^{F}-\pi_{1}^{N L} \geq 0$. From (3) and (7), we know that

$$
\Delta \pi_{1}=\pi_{1}^{F}-\pi_{1}^{N L}= \begin{cases}\frac{\theta^{2}}{(3-2 \beta)^{2}(2-\beta)^{2}} H_{1}(\beta) & \theta \leq \widehat{\theta}, \\ k-k_{2} & \theta>\widehat{\theta},\end{cases}
$$

where $H_{1}(\beta)=-2 \beta^{2}+4 \beta-1$ and $k_{2}=2(5+d-3 \beta)(1-d-$ $\beta) \theta^{2} /(3-2 \beta)^{2}(2+d-\beta)^{2}$. Then, if $\theta \leq \hat{\theta}$, making $\Delta \pi_{1} \geq 0$ only requires $H_{1}(\beta)=-2 \beta^{2}+4 \beta-1 \geq 0$. Substituting $\beta_{1}^{*} \in[0,1)$ into $H_{1}(\beta)=-2 \beta^{2}+4 \beta-1=0$, we have $\beta_{1}^{*}=(2-\sqrt{2}) / 2 \approx$ 0.293. If $\beta<\beta_{1}^{*}$, then $H_{1}(\beta)<0$; furthermore, we have $\Delta \pi_{1}<$ 0 . If $\beta>\beta_{1}^{*}$, then $H_{1}(\beta) \geq 0$. We also have $\Delta \pi_{1} \geq 0$.

What if $\theta>\hat{\theta}$ ? From (A.2), we know that $\beta>\beta_{2}^{*}=1-d$; then we have $\Delta \pi_{1} \geq 0$. However, if $\beta<\beta_{2}^{*}=1-d$, to make $\Delta \pi_{1} \geq 0$, we only have to consider the values of $k$ and $k_{2}$. Moreover, as $k<k_{1}=\theta^{2} /(2+d-\beta)^{2}$, we have to compare the values of $k_{1}$ and $k_{2}$ first. As

$$
k_{1}-k_{2}=\frac{\theta^{2}}{(2+d-\beta)^{2}(3-2 \beta)^{2}} H_{2}(\beta) \text {, }
$$

where $H_{2}(\beta)=-2 \beta^{2}+(1-d) \beta+2 d^{2}+8 d-1$; if $H_{2}(\beta)=$ $-2 \beta^{2}+(1-d) \beta+2 d^{2}+8 d-1=0$ is solvable, then $\Delta=17 d^{2}+$ $62 d-7 \geq 0$. As $d<d_{1}=(-32+6 \sqrt{30}) / 17 \approx 0.051, \Delta<0$, then $H_{2}(\beta)<0$; moreover, we have $k_{1}<k_{2}$. Then, we have $\Delta \pi_{1}<0$. 
If $d>d_{1}=(-32+6 \sqrt{30}) / 17 \approx 0.051$, then $\Delta \geq 0$, solving $H_{2}(\beta)=-2 \beta^{2}+(1-d) \beta+2 d^{2}+8 d-1=0$, and we have $\beta_{3}^{*}=\left((1-d)-\sqrt{17 d^{2}+62 d-7}\right) / 4$ and $\beta_{4}^{*}=((1-d)+$ $\left.\sqrt{17 d^{2}+62 d-7}\right) / 4$. To make $\beta_{3}^{*} \in[0,1-d)$, we only need $2 d^{2}+8 d-1 \leq 0$. Solving $2 d^{2}+8 d-1=0$ when $d \in[0,1]$, we have $d_{2}=(-4+3 \sqrt{2}) / 2 \approx 0.121$. Accordingly, when $d_{1} \leq d \leq$ $d_{2}$, then $0 \leq \beta_{3}^{*}<1-d$. Otherwise, $\beta_{3}^{*}<0$. To make $\beta_{4}^{*} \in$ $[0,1-d)$, we need $d^{2}+10 d-2 \leq 0$. We solve $d^{2}+10 d-2=0$ when $d \in[0,1]$, and thus we have $d_{3}=-5+3 \sqrt{3} \approx 0.196$. If $d_{1} \leq d \leq d_{3}$, then $0 \leq \beta_{4}^{*}<1-d$. Otherwise, $\beta_{4}^{*} \geq 1-d$.

Then, we have the following. (1) If $d_{1} \leq d \leq d_{2}$, then we have $0 \leq \beta_{3}^{*}<\beta_{4}^{*}<1-d$. In this case, if $\beta \leq \beta_{3}^{*}$ or $\beta \geq \beta_{4}^{*}$, we can derive that $H_{2}(\beta) \leq 0$ and $k_{1} \leq k_{2}$; if $\beta_{3}^{*}<\beta<\beta_{4}^{*}$, we have $H_{2}(\beta)>0$ and $k_{1}>k_{2}$. (2) If $d_{2}<d<d_{3}$, then we have $\beta_{3}^{*}<0$ and $0<\beta_{4}^{*}<1-d$. In this case, for any $\beta \in[0,1-d)$, if $\beta \leq \beta_{4}^{*}$, we can derive that $H_{2}(\beta) \geq 0$ and $k_{1} \geq k_{2}$; if $\beta>\beta_{4}^{*}$, we have $H_{2}(\beta)<0$ and $k_{1}<k_{2}$. (3) If $d \geq d_{3}$, we have $\beta_{3}^{*}<0$ and $\beta_{4}^{*} \geq 1-d$. In this case, for any $\beta \in[0,1-d)$, if $\beta<\beta_{4}^{*}$, we can derive that $H_{2}(\beta)>0$ and $k_{1}>k_{2}$.

To sum up the preceding proofs of $\theta>\hat{\theta}$, we have the following.

(1) If the product substitute rate is very small $\left(d<d_{1}=\right.$ $0.051)$, then regardless of R\&D cost of SP2, SP1 will choose not to implement fixed-fee licensing.

(2) If the product substitute rate is very small $\left(d_{1} \leq d \leq\right.$ $\left.d_{2}=0.121\right)$, then fixed-fee licensing will occur only if the network intensity is medium $\left(\beta_{3}^{*}<\beta<\beta_{4}^{*}\right)$ and $\mathrm{R} \& \mathrm{D}$ cost of SP2 is very high $\left(k_{2} \leq k<k_{1}\right)$.

(3) If the product substitute rate is medium $\left(d_{2}<d<\right.$ $\left.d_{3}=0.196\right)$, then fixed-fee licensing will occur only when the network intensity is very small $\left(\beta<\beta_{4}^{*}\right)$ and $\mathrm{R} \& \mathrm{D}$ cost of SP2 is very high $\left(k_{2} \leq k<k_{1}\right)$.

(4) If the product substitute rate is large enough $\left(d \geq d_{3}\right)$, then as long as R\&D cost of SP2 is very high $\left(k_{2} \leq k<\right.$ $\left.k_{1}\right)$, SP1 will choose to implement fixed-fee licensing.

Proof of Theorem 2. When $\theta \leq \widehat{\theta}$, we know that $r_{1}^{*} \leq r_{2}^{*}$. The optimal royalty rate is $r^{*}=\min \left\{r_{1}^{*}, r_{2}^{*}\right\}=r_{1}^{*}$. What about $\theta>\widehat{\theta}$ ? As $r_{1}^{*} \leq r_{2}^{*}$, we have the following:

$$
\theta \leq \widetilde{\theta}=\frac{2(2+d-\beta)\left(5-5 \beta+\beta^{2}\right) \sqrt{k}}{\sqrt{\left[10+(d-8) \beta+\beta^{2}\right]\left[10-(12+d) \beta+3 \beta^{2}\right]}} ;
$$

if $r_{1}^{*}>r_{2}^{*}$, then $\theta>\widetilde{\theta}$. This circumstance is also caused by $\tilde{\theta}-\hat{\theta}$

$$
=\frac{(2+d-\beta) \sqrt{k}}{\sqrt{\left[10+(d-8) \beta+\beta^{2}\right]\left[10-(12+d) \beta+3 \beta^{2}\right]}} H_{3}(\beta),
$$

$$
\begin{aligned}
& \text { where } H_{3}(\beta)=2(5-5 \beta \\
& \sqrt{\left[10+(d-8) \beta+\beta^{2}\right]\left[10-(12+d) \beta+3 \beta^{2}\right]}
\end{aligned}
$$

Through proof by contradiction, we have $\mathrm{H}_{3}(\beta)>0 \Rightarrow$ $\beta^{2}[\beta-(d+2)]^{2}>0$. Moreover, $\beta^{2}[\beta-(d+2)]^{2}>0$ is valid, so we have $H_{3}(\beta)>0 \Rightarrow \widetilde{\theta}-\widehat{\theta}>0$. Thus, $\widehat{\theta}<\theta \leq \widetilde{\theta}, r_{1}^{*}<r_{2}^{*}$. In other words, $r^{*}=\min \left\{r_{1}^{*}, r_{2}^{*}\right\}=r_{1}^{*}$, if $\theta>\widetilde{\theta}, r_{1}^{*}>r_{2}^{*}$. Thus, $r^{*}=\min \left\{r_{1}^{*}, r_{2}^{*}\right\}=r_{2}^{*}$. To sum up the conclusions under $\theta \leq \widehat{\theta}$ and $\theta>\widehat{\theta}$, we have Theorem 2 .

Proof of Theorem 3. When $\theta \leq \widehat{\theta}$, from (3) and (14), we have

$$
\pi_{1}^{R}-\pi_{1}^{N L}=\frac{\beta^{2} \theta^{2}}{4\left(5-5 \beta+\beta^{2}\right)(2-\beta)^{2}} \geq 0 .
$$

Therefore, SP1 may choose royalty licensing.

When $\widehat{\theta}<\theta \leq \widetilde{\theta}$, from (3) and (14), we have

$$
\begin{aligned}
\pi_{1}^{R}-\pi_{1}^{N L}= & \frac{\theta^{2}}{4\left(5-5 \beta+\beta^{2}\right)(2+d-\beta)^{2}} \\
& \times\left(\beta^{2}-10 d \beta+5 d^{2}+20 d\right)>0 .
\end{aligned}
$$

Under this circumstance, SP1 will implement royalty licensing instead of no licensing.

However, if $\theta>\widetilde{\theta}$,

$$
\begin{aligned}
\pi_{1}^{R}- & \pi_{1}^{N L} \\
= & \left(C_{1}(\beta) \theta^{2}+\left(5-5 \beta+\beta^{2}\right)(2+d-\beta)^{2} k\right. \\
& \left.+\theta \beta(2+d-\beta)^{2} \sqrt{\frac{\theta^{2}}{(2+d-\beta)^{2}}-k}\right) \\
& \times\left((2-\beta)^{2}(2+d-\beta)^{2}\right)^{-1},
\end{aligned}
$$

where $C_{1}(\beta)=-\beta^{2}+(5-2 d) \beta+d^{2}+4 d-5$. From this equation, we know that if $C_{1}(\beta) \geq 0 \Rightarrow \pi_{1}^{R}>\pi_{1}^{N L}$, then solving $C_{1}(\beta)=$ 0 when $\beta \in[0,1)$ gives us $\beta_{5}^{*}=\left(5-2 d-\sqrt{5-4 d+8 d^{2}}\right) / 2$ and $\beta_{6}^{*}=\left(5-2 d+\sqrt{5-4 d+8 d^{2}}\right) / 2$. Clearly, $\beta_{6}^{*}>1$. Moreover, $0 \leq \beta_{5}^{*}<1 \Rightarrow d^{2}+2 d-1>0$, whereas $d>d_{4}=\sqrt{2}-1 \approx$ 0.414. We have $d^{2}+2 d-1>0$; $d<d_{4}$. Thus, $d^{2}+2 d-1 \leq 0$.

Then, we have that when $d>d_{4}=\sqrt{2}-1 \approx 0.414, \beta_{6}^{*}>$ land $0 \leq \beta_{5}^{*}<1$. If $\beta \geq \beta_{5}^{*}$, then $C_{1}(\beta) \geq 0$; if $0 \leq \beta<\beta_{5}^{*}$, then $C_{1}(\beta)<0$. However, if $d \leq d_{4}, \beta_{6}^{*}>1$ and $\beta_{5}^{*} \geq 1$. For anywhere between $\beta \in[0,1), C_{1}(\beta)<0$. When $d>d_{4}$ and $\beta \geq \beta_{5}^{*}$, we have $\pi_{1}^{R}>\pi_{1}^{N L}$. Therefore, we only have to compare the values of $\pi_{1}^{R}$ and $\pi_{1}^{N L}$, when $d>d_{4}=\sqrt{2}-1 \approx$ 0.414 and $0 \leq \beta<\beta_{5}^{*}$ or $d \leq d_{4}$, indicating that $C_{1}(\beta)<0$.

When $C_{1}(\beta)<0$, if $\pi_{1}^{R}>\pi_{1}^{N L}, \theta \beta(2+d-$ $\beta)^{2} \sqrt{\theta^{2} /(2+d-\beta)^{2}-k} \geq C_{2}(\beta)$, where $C_{2}(\beta)=\left[\beta^{2}-(5-\right.$ 2d) $\left.\beta-d^{2}-4 d+5\right] \theta^{2}-\left(\beta^{2}-5 \beta+5\right)(2+d-\beta)^{2} k$. 
Let

$$
\begin{gathered}
k_{4}=\frac{\beta^{2}-(5-2 d) \beta-d^{2}-4 d+5}{\left(5-5 \beta+\beta^{2}\right)(2+d-\beta)^{2}} \theta^{2}, \\
\text { as } \quad k_{1}-k_{4}=\frac{d(d+4-2 \beta)}{\left(5-5 \beta+\beta^{2}\right)(2+d-\beta)^{2}} \theta^{2} \geq 0 .
\end{gathered}
$$

We have $C_{2}(\beta) \leq 0$ when $k \leq k_{4}$; thus, $\pi_{1}^{R}>\pi_{1}^{N L}$. However, if $k_{4}<k<k_{1}$, we have $C_{2}(\beta)>0$. We then continue to compare the values of $\pi_{1}^{R}$ and $\pi_{1}^{N L}$ when $C_{2}(\beta)>0$, and

$$
\begin{aligned}
& \theta \beta(2+d-\beta)^{2} \sqrt{\frac{\theta^{2}}{(2+d-\beta)^{2}}-k} \geq C_{2}(\beta) \\
& \Longrightarrow C_{3} k^{2}+C_{4} k+C_{5} \leq 0,
\end{aligned}
$$

where

$$
\begin{aligned}
C_{3}=(5 & \left.-5 \beta+\beta^{2}\right)^{2}(2+d-\beta)^{4}, \\
C_{4}= & {\left[\beta^{2}(2+d-\beta)^{2}\right.} \\
& \left.-2\left(\beta^{2}-(5-2 d) \beta-d^{2}-4 d+5\right)\left(5-5 \beta+\beta^{2}\right)\right] \\
& \times(2+d-\beta)^{2} \theta^{2}, \\
C_{5}= & {\left[\left(\beta^{2}-(5-2 d) \beta-d^{2}-4 d+5\right)^{2}\right.} \\
& \left.-\beta^{2}(2+d-\beta)^{2}\right] \theta^{4} .
\end{aligned}
$$

Solving $C_{3} k^{2}+C_{4} k+C_{5}=0$ yields two results: one is smaller than 0 and the other is $k_{3}=\left(-C_{4}+\sqrt{\Delta_{1}}\right) / 2 C_{3}$. If $k_{3}>$ 0 , we will have $C_{3} C_{5}<0$. As $\beta \geq \beta_{2}^{*}=1-d, C_{3}>0, C_{5}>0$, $\beta<\beta_{2}^{*}, C_{3}>0$, and $C_{5}<0$, we have $\beta \geq \beta_{2}^{*}=1-d$ and $k_{3} \leq 0 \Rightarrow C_{3} k^{2}+C_{4} k+C_{5}>0 \Rightarrow \pi_{1}^{R}<\pi_{1}^{N L}$. However, if $\beta<\beta_{2}^{*}, k_{3}>0$, we have

$$
\begin{aligned}
& k_{3} \leq k_{1} \\
& \Longrightarrow\left\{\begin{array}{l}
k<k_{3} \Longrightarrow C_{3} k^{2}+C_{4} k+C_{5} \leq 0 \Longrightarrow \pi_{1}^{R} \geq \pi_{1}^{N L}, \\
k_{3}<k<k_{1} \Longrightarrow C_{3} k^{2}+C_{4} k+C_{5}>0 \Longrightarrow \pi_{1}^{R}<\pi_{1}^{N L},
\end{array}\right. \\
& k_{3}>k_{1} \Longrightarrow k<k_{1} \Longrightarrow C_{3} k^{2}+C_{4} k+C_{5}<0 \Longrightarrow \pi_{1}^{R}>\pi_{1}^{N L} .
\end{aligned}
$$

The only issue left is determining the conditions in which $k_{3}$ can be located between the lower limit of $k_{4}$ and the upper limit of $k_{1}$.

Then, we have $k_{4}-k_{3}=\left(\beta^{2}(2+d-\beta)^{4} \theta^{2}-\sqrt{\Delta_{1}}\right) / 2 C_{3} \leq$ $0 \Rightarrow d(2 \beta-d-4) \leq 0$, and $d(2 \beta-d-4) \leq 0$ is valid under $d \in[0,1]$. Accordingly, $k_{4}-k_{3} \leq 0$. In other words, $k_{4}$ is the lower limit of $k_{3}$, and

$$
\begin{aligned}
& k_{3}-k_{1}=\frac{-C_{4}+\sqrt{\Delta_{1}}}{2 C_{3}}-\frac{\theta^{2}}{(2+d-\beta)^{2}} \leq 0 \\
& \Longrightarrow \sqrt{\Delta_{1}} \leq \frac{2 C_{3}}{(2+d-\beta)^{2}} \theta^{2}+C_{4} .
\end{aligned}
$$

We can easily derive that

$$
\beta_{7}^{*}=\frac{-2 d^{3}-16 d^{2}+\sqrt{\Delta_{2}}}{2\left(4 d^{2}-40 d+50\right)} .
$$

Moreover, $\beta_{7}^{*}<1 \Rightarrow 3 d^{3}-2 d^{2}+20 d-25<0$, and $3 d^{3}-$ $2 d^{2}+20 d-25<0$ is valid under $d \in[0,1]$; therefore $\beta_{7}^{*}<1$ is valid. We have $\beta \leq \beta_{7}^{*} \Rightarrow k_{3} \leq k_{1}, \beta>\beta_{7}^{*} \Rightarrow k_{3}>k_{1}$. Then, we compare the value of $\beta_{7}^{*}$ and the critical value of $\beta_{2}^{*}$. After complex deduction, we have $\beta_{7}^{*}<\beta_{2}^{*} \Rightarrow C_{7}(d)<0$, where $C_{7}(d)=-2 d^{6}+90 d^{5}-859 d^{4}+3030 d^{3}-3125 d^{2}+2250 d-$ 625. Through MATLAB, we obtain $0 \leq d \leq d_{5} \approx 0.314$. After solving the equation, we have $C_{7}(d) \leq 0 \Rightarrow \beta_{7}^{*}<\beta_{2}^{*}$. Otherwise, $C_{7}(d)>0 \Rightarrow \beta_{7}^{*}>\beta_{2}^{*}$. We compare the values of $\beta_{5}^{*}$ and $\beta_{2}^{*}$ under $d>d_{4}=\sqrt{2}-1 \approx 0.414$ and $0 \leq \beta<$ $\beta_{5}^{*}$. Consider $\beta_{5}^{*}-\beta_{2}^{*} \geq 0 \Rightarrow 2 d^{2}-d-1 \leq 0$. Moreover, $2 d^{2}-d-1 \leq 0$ is invariably valid under $d \in[0,1]$. As a result, we have $\beta_{5}^{*} \geq \beta_{2}^{*}$.

To sum up the conclusions under $\theta>\widetilde{\theta}$, we have the following.

(1) If the product substitute rate is very high $\left(d>d_{4}=\right.$ $\sqrt{2}-1 \approx 0.414)$ and the network is large enough $(\beta>$ $\left.\beta_{5}^{*}\right), \mathrm{SP} 1$ is motivated to implement royalty licensing.

(2) When the product substitute rate is very high $(d>$ $\left.d_{4}\right)$, but the network intensity is not large enough $\left(\beta<\beta_{5}^{*}\right)$, the following three situations may occur. (1) If the network intensity is medium $\left(1-d=\beta_{2}^{*} \leq\right.$ $\beta<\beta_{5}^{*}$ ), regardless of the R\&D cost for SP2, SP1 will not choose to implement royalty licensing. (2) If the network intensity is very small $\left(\beta<\beta_{2}^{*}\right)$, and if $R \& D$ cost of SP2 is very low $\left(k_{4}<k<k_{3}\right)$, then royalty licensing will occur. If R\&D cost of SP2 is very high $\left(k_{3} \leq k<k_{1}\right)$, then SP1 will choose not to implement royalty licensing. (3) As long as R\&D cost of SP2 is low enough $\left(k \leq k_{4}\right)$, SP1 has the motivation to implement royalty licensing.

(3) When the product substitute rate is not very high ( $d \leq$ $d_{4}$ ), the licensing conditions are as follows. (a) If the network intensity is very large $\left(\beta \geq \beta_{2}^{*}\right)$, regardless of the R\&D cost of SP2, SP1 will not choose to implement royalty licensing. (b) If the product substitute rate is medium $\left(0.314 \approx d_{5}<d \leq d_{4}\right)$, network intensity is not very large $\left(\beta<\beta_{2}^{*}\right)$ and R\&D cost of SP2 is very high $\left(k_{3} \leq k<k_{1}\right)$; SP1 will choose not to implement royalty licensing; if R\&D cost of SP2 is very low $\left(k_{4}<\right.$ $k<k_{3}$ ), royalty licensing will occur. (c) If the product substitute rate is very low $\left(0 \leq d \leq d_{5}\right)$ and network intensity is very small $\left(\beta_{7}^{*}<\beta<\beta_{2}^{*}\right)$, royalty licensing will occur. (d) If the product substitute rate is very low $\left(0 \leq d \leq d_{5}\right)$, network intensity is very small $(\beta<$ $\left.\beta_{7}^{*}\right)$ and R\&D cost of SP2 is very high $\left(k_{3} \leq k<k_{1}\right)$; SP1 will choose not to implement royalty licensing; if $\mathrm{R} \& \mathrm{D}$ cost of SP2 is very small $\left(k_{4}<k<k_{3}\right)$, royalty licensing will occur. (e) As long as R\&D cost of SP2 is 
low enough $\left(k \leq k_{4}\right)$, SP1 is motivated to implement royalty licensing.

Proof of Proposition 4. When $\theta \leq \widehat{\theta}, \pi_{1}^{F}-\pi_{1}^{R}=\left(\left(-12 \beta^{2}+\right.\right.$ $\left.20 \beta-5) / 4(3-2 \beta)^{2}\left(5-5 \beta+\beta^{2}\right)\right) \theta^{2}$, for $-12 \beta^{2}+20 \beta-5=0$, if one root is $\beta_{8}^{*}=(5-\sqrt{10}) / 6 \approx 0.306$ under $\beta \in[0,1)$ (the other root is $(5+\sqrt{10}) / 6>1)$ and if $\beta<\beta_{8}^{*}$, then $\pi_{1}^{R}>\pi_{1}^{F}$; if $\beta>\beta_{8}^{*}$, then $\pi_{1}^{F}>\pi_{1}^{R}$. Moreover, $\beta_{1}^{*} \leq \beta$ and $\beta_{1}^{*} \leq \beta_{8}^{*}$. Case (1) in Proposition 4 is obtained.

When

$$
\begin{gathered}
\hat{\theta}<\theta \leq \tilde{\theta}, \\
\pi_{1}^{F}-\pi_{1}^{R}=k-k_{2}-\frac{\beta^{2}-10 d \beta+20 d+5 d^{2}}{4(2+d-\beta)^{2}\left(5-5 \beta+\beta^{2}\right)} \theta^{2} \\
=k-k_{5},
\end{gathered}
$$

where

$$
k_{5}=k_{2}+\frac{\beta^{2}-10 d \beta+20 d+5 d^{2}}{4(2+d-\beta)^{2}\left(5-5 \beta+\beta^{2}\right)} \theta^{2}
$$

Then, we know that when $k>k_{5}$, we have $\pi_{1}^{F}>\pi_{1}^{R}$; when $k<k_{5}$, we have $\pi_{1}^{R}>\pi_{1}^{F}$. As $k<k_{1}$, we have to ensure $k_{5}<k_{1}$ to keep the conclusion valid. As indicated in Figure 5, we prove the existence of $\beta_{9}^{*} \approx 0.319$, which makes $k_{5}=k_{1}$, through MATLAB. When $\beta<\beta_{9}^{*}$, we have $k_{1}<k_{5}$ and when $\beta>\beta_{9}^{*}$, we have $k_{1}>k_{5}$.

After considering all four situations under fixed-fee licensing, we obtain the conclusions in the case of $\widehat{\theta}<\theta \leq \tilde{\theta}$ as follows.

(a) If the network intensity is strong enough $(\beta \geq$ $\left.\max \left\{\beta_{2}^{*}, \beta_{9}^{*}\right\}\right)$ and if SP2's R\&D cost is extremely high $\left(k_{5} \leq\right.$ $k<k_{1}$ ), then fixed-fee licensing will be better than royalty licensing. However, if SP2's R\&D cost is extremely low $\left(k<k_{5}\right)$, then royalty licensing will be better than fixed-fee licensing. (b) If the product substitution rate is extremely high $\left(d \geq d_{3}\right)$ but the network intensity is not high enough $(\beta<$ $\left.\max \left\{\beta_{2}^{*}, \beta_{9}^{*}\right\}\right)$ or if the product substitution rate is medium $\left(d_{2}<d<d_{3}\right)$ and the network intensity is extremely low $(\beta \leq$ $\max \left\{\beta_{4}^{*}, \beta_{9}^{*}\right\}$ ), for any $k<k_{1}$, then royalty licensing will be better than fixed-fee licensing. (c) If the product substitution rate is extremely small $\left(d_{1} \leq d \leq d_{2}\right)$, the network intensity is medium $\left(\max \left\{\beta_{3}^{*}, \beta_{9}^{*}\right\}<\beta<\min \left\{\beta_{4}^{*}, \beta_{9}^{*}\right\}\right)$ and SP2's $\mathrm{R} \& \mathrm{D}$ cost is extremely high; then royalty licensing will be better than fixed-fee licensing. If SP2's R\&D cost is extremely small $\left(k_{2} \leq k<k_{5}\right)$, then royalty licensing will be better than royalty licensing. If SP2's R\&D cost is extremely high, then royalty licensing will be better than fixed-fee licensing. If SP2's $\mathrm{R} \& \mathrm{D}$ cost is extremely small $\left(k_{2} \leq k<k_{5}\right)$, then royalty licensing will be better than royalty licensing.

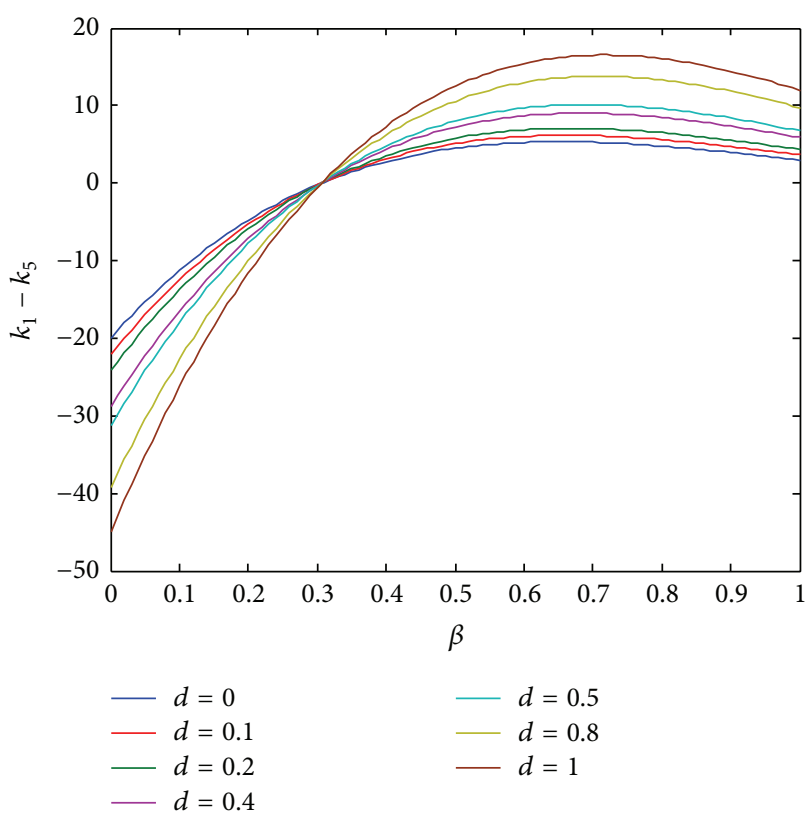

FIGURE 5: Varying patterns of $k_{1}-k_{5}$ under different values of $d$ regarding $\beta$.

When $\theta>\tilde{\theta}$,

$$
\begin{aligned}
\pi_{1}^{F}- & \pi_{1}^{R} \\
= & \left(H_{4} \theta^{2}-(3-2 \beta)^{2}(2+d-\beta)^{2}\right. \\
& \times\left(\theta \beta \sqrt{\left.\left.\frac{\theta^{2}}{(2+d-\beta)^{2}}-k+(1-\beta) k\right)\right)}\right. \\
& \times\left((3-2 \beta)^{2}(2+d-\beta)^{2}(2-\beta)^{2}\right)^{-1},
\end{aligned}
$$

where $H_{4}=\left[-2 \beta^{2}+(4-6 d) \beta+2 d^{2}+8 d-1\right](2-\beta)^{2}-[(1-2 d) \beta+$ $\left.d^{2}+4 d-1\right](3-2 \beta)^{2}$. As indicated in Figure 6 , we invariably have $H_{4}>0$ anywhere between intervals $\beta \in[0,1)$.

Let $k_{6}=\left(H_{4} /(2+d-\beta)^{2}(3-2 \beta)^{2}(1-\beta)\right) \theta^{2}$. Thus, we have $k_{6}>0$. As a result, if $k>k_{6}$, we have $\pi_{1}^{R}>\pi_{1}^{F}$. To make $\pi_{1}^{R}>\pi_{1}^{F}$, we must have $k \leq k_{6}$ and $H_{5}=(1-\beta)\left(k_{6}-k\right)-$ $\theta \beta \sqrt{\theta^{2} /(2+d-\beta)^{2}-k} \geq 0$. As $H_{5}$ is the quadratic curve of $k$, with its open upward and its discriminant as $\Delta_{3} \leq 0$, where $\Delta_{3}=\left[\beta^{2}-2(1-\beta) H_{4}\right]^{2} \theta^{4}-4(1-\beta)^{2}\left[H_{4}-\beta^{2} /(2+d-\beta)^{2}\right] \theta^{4}$, as indicated in Figure 7, then $H_{5} \geq 0$ is invariably valid. Thus, if $k \leq k_{6}$, we have $\pi_{1}^{F} \geq \pi_{1}^{R}$. Considering the aforementioned 15 possible combinations, we have to compare the value of $k_{6}$ and these critical values and make comparisons within the critical values themselves.

In the following, we first compare $k_{2}$ with $k_{6}$.

$k_{2}-k_{6}=2 \beta^{4}-(14+2 d) \beta^{3}+\left(2 d^{2}+4 d+31\right) \beta^{2}-\left(4 d^{2}+\right.$ $6 d+15) \beta-d^{2}+4 d+5$, as indicated in Figure 8 . As anywhere between interval $\beta \in[0,1), k_{2}>k_{6}$ is invariably valid. 


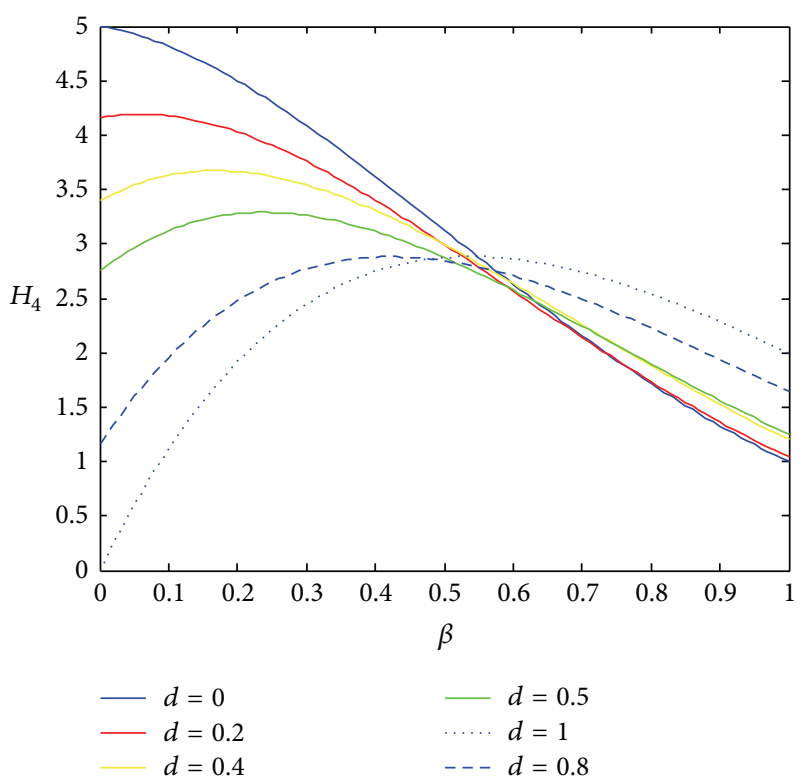

FIGURE 6: Varying patterns of $H_{4}$ under different values of $d$ regarding $\beta$.

We then compare the critical values of $k_{2}$ and $k_{3}$. Consider that $k_{2}-k_{3} \leq 0 \Rightarrow C_{3} k_{2}^{2}+C_{4} k_{2}+C_{5} \leq 0$ as indicated in Figure 9 because $C_{3} k_{2}^{2}+C_{4} k_{2}+C_{5}$ is invariably smaller than 0 under different values of $d$ regarding $\beta \in[0,1)$. Therefore, $k_{2} \leq k_{3}$.

We then compare the critical values of $k_{4}$ and $k_{6}$ under conditions $d>d_{4}$ and $\beta_{2}^{*} \leq \beta<\beta_{5}^{*}$ or $d \leq d_{4}$ and $\beta \geq \beta_{2}^{*}$.

As

$$
k_{4}-k_{6}=\frac{H_{6}}{\left(5-5 \beta+\beta^{2}\right)(2+d-\beta)^{2}(3-2 \beta)^{2}(1-\beta)} \theta^{2},
$$

where $H_{6}=(1-\beta)(3-2 \beta)^{2}\left[\beta^{2}-(5-2 d) \beta-d^{2}-4 d+5\right]-$ $H_{4}\left(5-5 \beta+\beta^{2}\right)$, as indicated in Figure 10 , then $k_{4} \leq k_{6}$ is invariably valid under $d>d_{4}$ and $\beta_{2}^{*} \leq \beta<\beta_{5}^{*}$ or $d \leq d_{4}$ and $\beta \geq \beta_{2}^{*}$. Thus, we have $k_{3} \geq k_{2}>k_{6}>k_{4}$ between intervals of $d \in[0,0.414]$.

The only task left is to compare the critical values of $\beta_{4}^{*}$ and $\beta_{7}^{*}$. Moreover,

$$
\beta_{4}^{*}-\beta_{7}^{*}=\frac{H_{7}}{4\left(2 d^{2}-20 d+25\right)},
$$

where

$$
\begin{aligned}
H_{7}= & 38 d^{2}-45 d+25 \\
& +\left(2 d^{2}-20 d+25\right) \sqrt{17 d^{2}+62 d-7}-\sqrt{\Delta_{2}}, \\
\Delta_{2}= & 4 d^{2}\left[(d+8)^{2}+(8 d+16)\left(4 d^{2}-40 d+50\right)\right] .
\end{aligned}
$$

As indicated in Figure 11, $H_{7}$ is a decreasing function between $d \in[0,0.414]$ regarding $d$ and $H_{7}<0$. Thus, we have $\beta_{4}^{*}<\beta_{7}^{*}$.

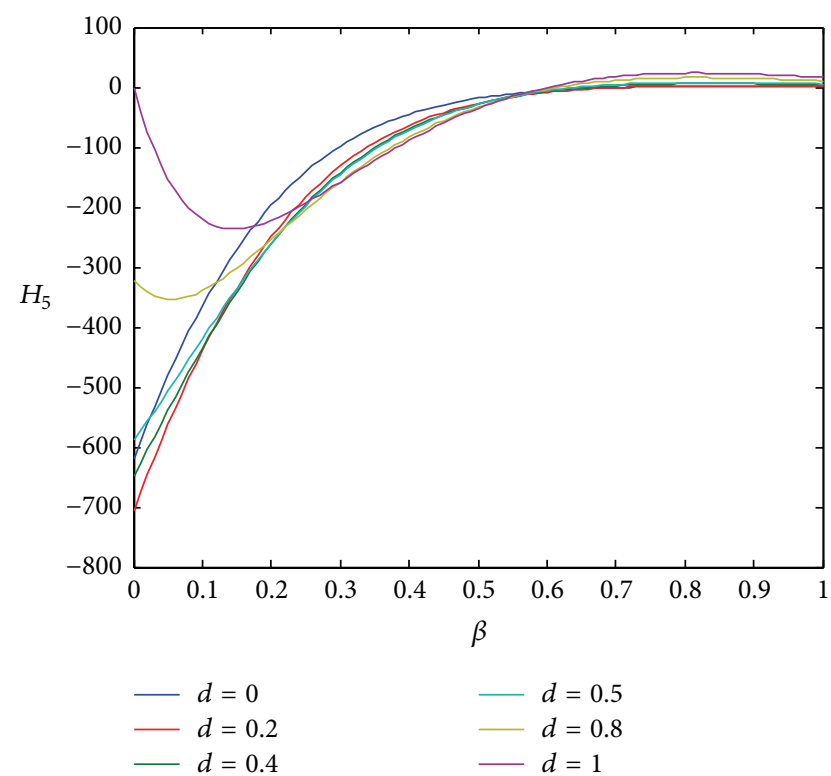

FIGURE 7: Varying patterns of $H_{5}$ under different values of $d$ regarding $\beta$.

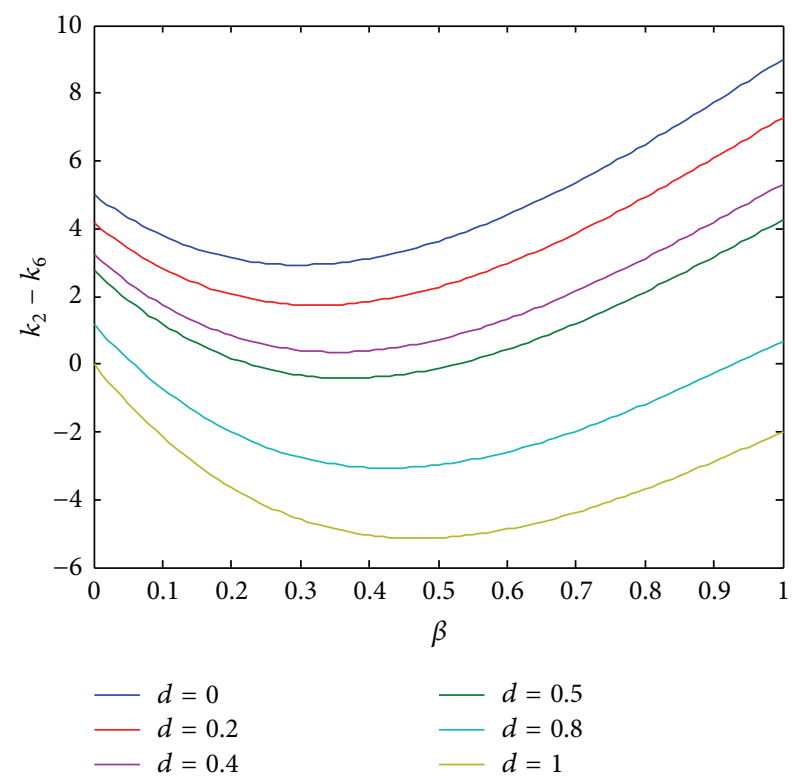

FIGURE 8: Varying patterns of $k_{2}-k_{6}$ under different values of $d$ regarding $\beta$.

After considering 15 possible combinations, we have eight situations in the case of $\theta>\widetilde{\theta}$.

(a) If the product substitution rate is extremely high $(d>$ $\left.d_{4}\right)$, the network intensity is extremely high $\left(\beta \geq \beta_{5}^{*}\right)$ and SP2's R\&D cost is extremely high $\left(k_{6}<k<k_{1}\right)$; then royalty licensing will be better than fixed-fee licensing. However, if SP2's R\&D cost is extremely small $\left(k<k_{6}\right)$, then fixedfee licensing will be better than royalty licensing. (b) If the product substitution rate is extremely high $\left(d>d_{4}\right)$ but the network intensity is medium $\left(\beta_{2}^{*} \leq \beta<\beta_{5}^{*}\right)$, then it will 


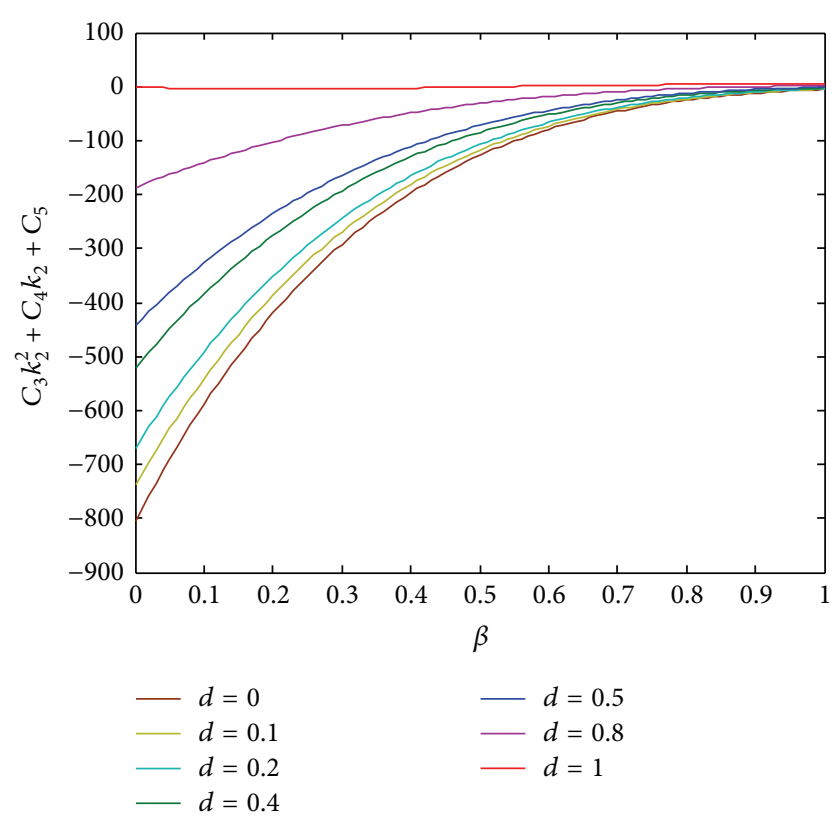

Figure 9: Varying patterns of $C_{3} k_{2}^{2}+C_{4} k_{2}+C_{5}$ under different values of $d$ regarding $\beta$.

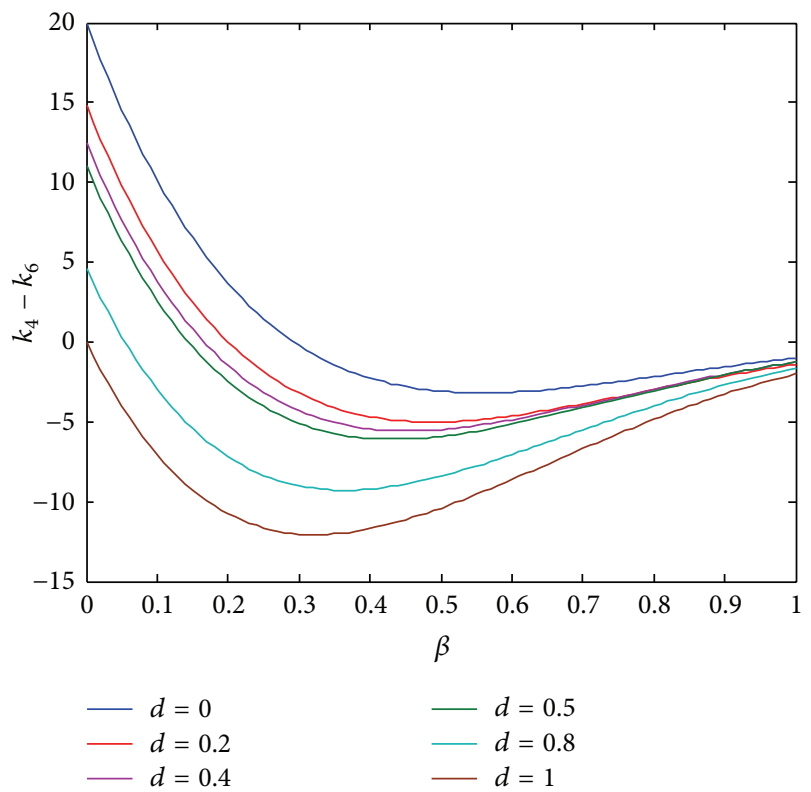

FIGURE 10: Varying patterns of $k_{4}-k_{6}$ under different values of $d$ regarding $\beta$.

always be true that fixed-fee licensing is better than royalty licensing for SP2 when it has small R\&D cost $\left(k \leq k_{4}\right)$. (c) If the product substitution rate is not high $\left(d \leq d_{4}\right)$ and the rate of network intensity is extremely high $\left(\beta \geq \beta_{2}^{*}\right)$, it will always be true that fixed-fee licensing is better than royalty licensing for any R\&D cost $\left(k \leq k_{4}\right)$ for SP2. (d) If the product substitution rate is extremely small $\left(d_{3} \leq d \leq d_{5}\right)$ and the network intensity is medium $\left(\beta_{7}^{*}<\beta<\beta_{2}^{*}\right)$, then royalty licensing will be better than fixed-fee licensing for any $R \& D$

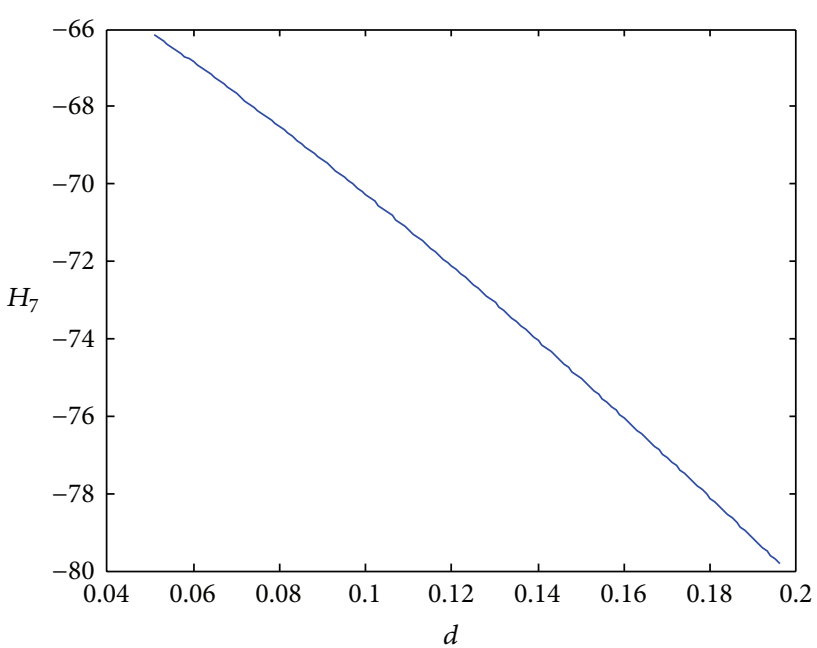

FIGURE 11: Varying patterns of $H_{7}$ regarding $d$.

cost $\left(k_{2} \leq k \leq k_{1}\right)$ for SP2. (e) If the product substitution rate is extremely high $\left(d>d_{5}\right)$, the network intensity is relevantly small $\left(\beta<\beta_{2}^{*}\right)$ and SP2's R\&D cost is medium $\left(k_{2} \leq k<k_{3}\right)$; then royalty licensing will be better than fixedfee licensing. (f) If the product substitution rate is medium $\left(d_{3} \leq d \leq d_{5}\right)$, the network intensity is relevantly low $(\beta<$ $\left.\beta_{7}^{*}\right)$ and SP2's R\&D cost is medium $\left(k_{2} \leq k<k_{3}\right)$; then royalty licensing will be better than fixed-fee licensing. (g) If the product substitution rate is extremely low $\left(d_{2}<d<d_{3}\right)$, the network intensity is extremely low $\left(\beta<\beta_{4}^{*}\right)$ and SP2's R\&D cost is medium $\left(k_{2} \leq k<k_{3}\right)$; then royalty licensing will be better than fixed-fee licensing. (h) If the product substitution rate is extremely low $\left(d_{1} \leq d \leq d_{2}\right)$, the network intensity is extremely low $\left(\beta_{3}^{*}<\beta<\beta_{4}^{*}\right)$ and SP2's R\&D cost is medium $\left(k_{2} \leq k<k_{3}\right)$; then royalty licensing will be better than fixedfee licensing.

\section{Conflict of Interests}

The authors declare that there is no conflict of interests regarding the publication of this paper.

\section{Acknowledgments}

The authors would like to thank the editor and anonymous referees for their insightful comments and suggestions that have significantly improved this paper. Xianpei Hong's research is partially supported by Humanity and Social Science Youth Foundation of Ministry of Education of China, Grant no. 11YJC630058, the Fundamental Research Funds for the Central Universities, Program no. 2662014BQ048, and Research Project of Department of Education of Hubei Province, Grant no. Q20141803. Haiqing Hu's research is partially supported by Humanities and Social Sciences Foundation of Ministry of Education in China, Grant no. 14YJC630049. 


\section{References}

[1] P. Germerad, “The changing role of R\&D," Reach-Technology Management, vol. 44, pp. 15-20, 2001.

[2] P. C. Grindley and D. J. Teece, "Managing intellectual capital: licensing and cross-licensing in semiconductors and electronics," California Management Review, vol. 39, no. 2, pp. 8-41, 1997.

[3] M. L. Katz and C. Shapiro, "Systems competition and network effects," Journal of Economic Perspectives, vol. 8, no. 2, pp. 93-115, 1994.

[4] Y. Chen and J. Xie, "Cross-market network effect with asymmetric customer loyalty: implications for competitive advantage," Marketing Science, vol. 26, no. 1, pp. 52-66, 2007.

[5] M. I. Kamien, S. S. Oren, and Y. Tauman, "Optimal licensing of cost-reducing innovation," Journal of Mathematical Economics, vol. 21, no. 5, pp. 483-508, 1992.

[6] I. Macho-Stadler, X. Martinez-Giralt, and J. D. Pérez-Castrillo, "The role of information in licensing contract design," Research Policy, vol. 25, no. 1, pp. 43-57, 1996.

[7] A. Mukherjee and E. Pennings, "Tariffs, licensing and market structure," European Economic Review, vol. 50, no. 7, pp. 16991707, 2006.

[8] M. H. Nabin, X. N. Guyen, and P. M. Sgro, "Optimal licensing policy under vertical product differentiation," Working Paper, 2011.

[9] A. Shaked and J. Sutton, "Relaxing price competition through product differentiation," The Review of Economic Studies, vol. 49, no. 1, pp. 3-13, 1982.

[10] A. Shaked and J. Sutton, "Natural oligopolies," Econometrica, vol. 51, no. 5, pp. 1469-1483, 1983.

[11] A. Shaked and J. Sutton, "Involuntary unemployment as a perfect equilibrium in a bargaining model," Econometrica, vol. 52, no. 6, pp. 1351-1364, 1984.

[12] G. Stamatopoulos and Y. Tauman, "Licensing of a qualityimproving innovation," Mathematical Social Sciences, vol. 56, no. 3, pp. 410-438, 2008.

[13] C. Li and J. Wang, "Licensing a vertical product innovation," Economic Record, vol. 86, no. 275, pp. 517-527, 2010.

[14] B. Shen, T.-M. Choi, Y. Wang, and C. K. Y. Lo, "The coordination of fashion supply chains with a risk-averse supplier under the markdown money policy," IEEE Transactions on Systems, Man, and Cybernetics Part A: Systems and Humans, vol. 43, no. 2, pp. 266-276, 2013.

[15] C. Y. Li and X. Y. Geng, "Licensing to a durable-good monopoly," Economic Modelling, vol. 25, no. 5, pp. 876-884, 2008.

[16] M. I. Kamien and Y. Tauman, "Fees versus royalties and the private value of a patent," The Quarterly Journal of Economics, vol. 101, no. 3, pp. 471-491, 1986.

[17] S. Muto, "On licensing policies in Bertrand competition," Games and Economic Behavior, vol. 5, no. 2, pp. 257-267, 1993.

[18] P. Crama, B. de Reyck, and Z. Degraeve, "Milestone payments or royalties? Contract design for R\&D licensing," Operations Research, vol. 56, no. 6, pp. 1539-1552, 2008.

[19] A. Mukherjee, "Licensing a new product: fee vs. royalty licensing with unionized labor market," Labour Economics, vol. 17, no. 4, pp. 735-742, 2010.

[20] L. Lin and N. Kulatilaka, "Network effects and technology licensing with fixed fee, royalty, and hybrid contracts," Journal of Management Information Systems, vol. 23, no. 2, pp. 91-118, 2006.
[21] T. M. Choi, P. S. Chow, and G. Kannan, "Service optimization and control," Mathematical Problems in Engineering, vol. 2014, Article ID 951376, 3 pages, 2014.

[22] C. H. Chiu, T. M. Choi, Y. Li, and L. Xu, "Service competition and service war: a game-theoretic analysis," Service Science, vol. 6, no. 1, pp. 63-76, 2014.

[23] W. K. Chan and B. J. Gao, "Unfair consequence of fair competition in service systems - an agent-based and queuing approach," Service Science, vol. 5, no. 3, pp. 249-262, 2013.

[24] Y. Li, L. Xu, and D. Li, "Examining relationships between the return policy, product quality, and pricing strategy in online direct selling," International Journal of Production Economics, vol. 144, no. 2, pp. 451-460, 2013.

[25] L. Xu, Z. Wang, J. Shan, and Y. Wang, "On advertising games and spillover in service systems," Mathematical Problems in Engineering, vol. 2013, Article ID 260823, 8 pages, 2013.

[26] G. F. Gilder, Telecoms: How Infinite Bandwidth Will Revolutionize Our World, Free Press, New York, NY, USA, 2000.

[27] M. L. Katz and C. Shapiro, "On the licensing of innovations," The RAND Journal of Economics, vol. 16, no. 4, pp. 504-520, 1985. 


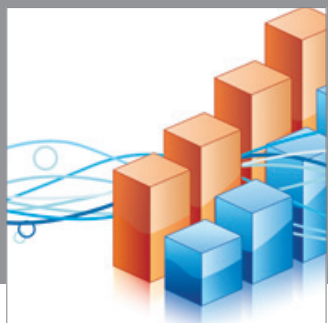

Advances in

Operations Research

mansans

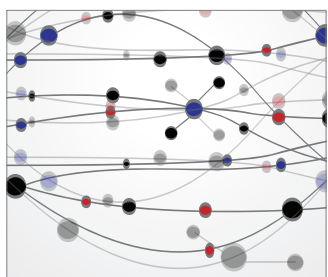

The Scientific World Journal
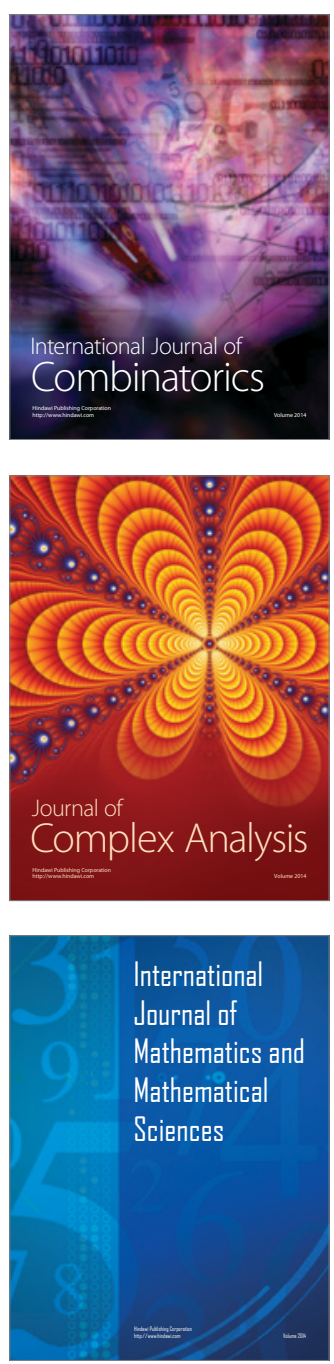
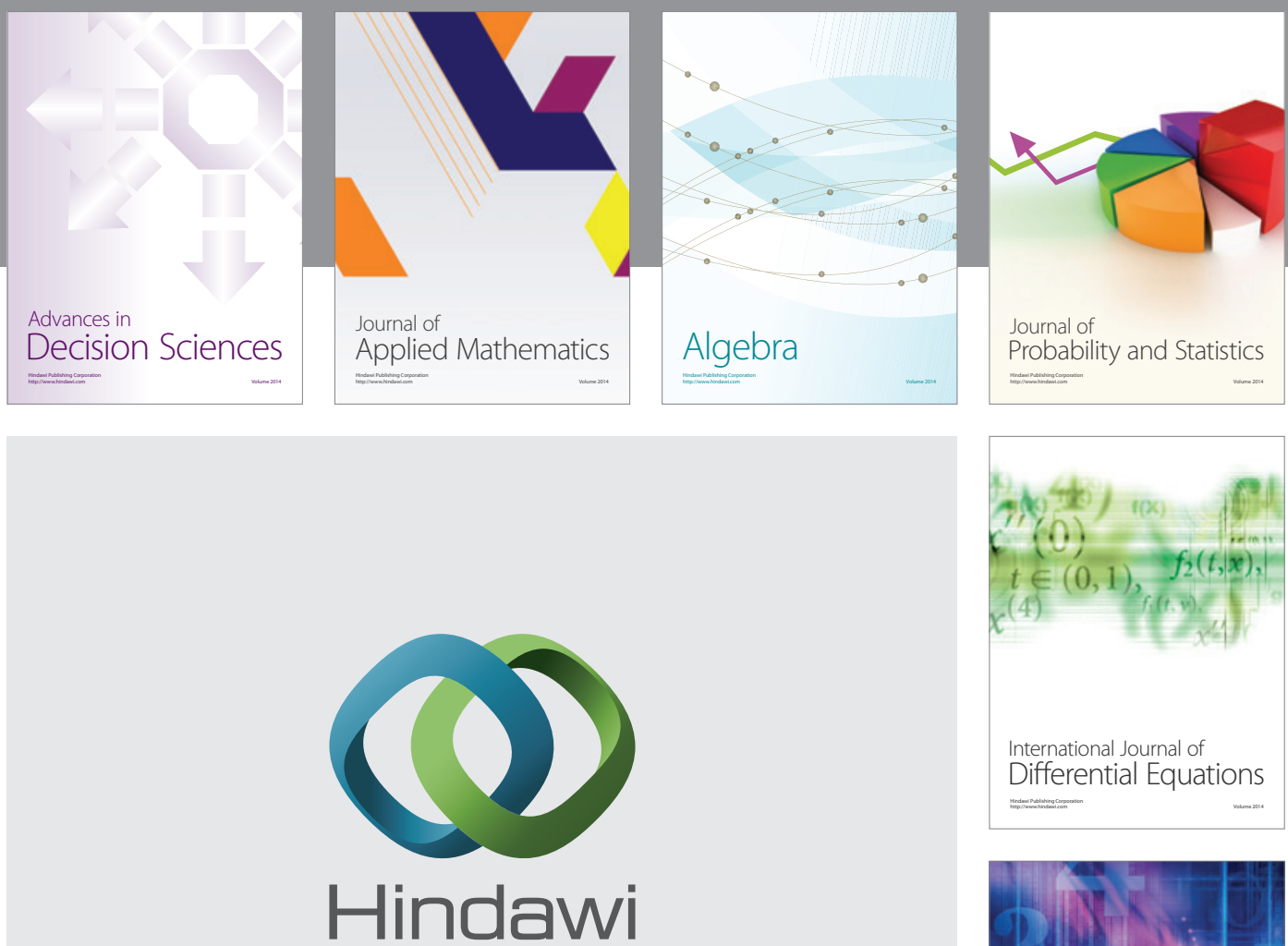

Submit your manuscripts at http://www.hindawi.com
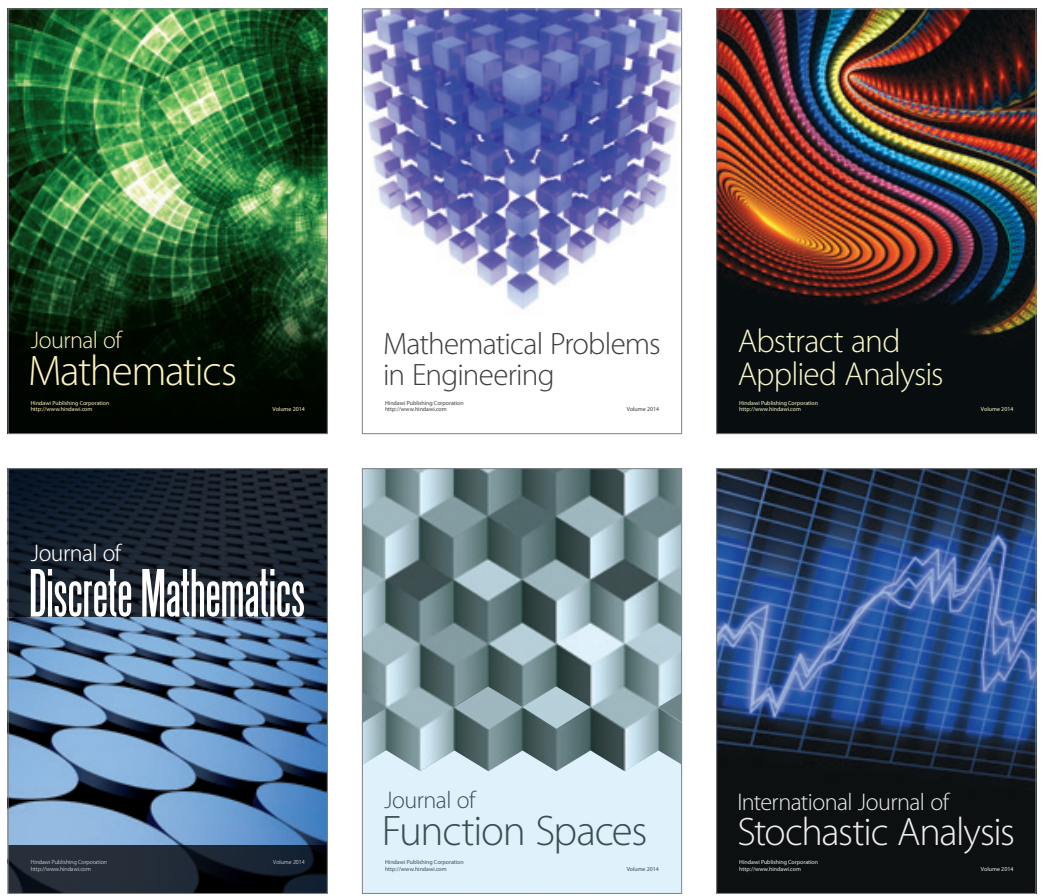

Journal of

Function Spaces

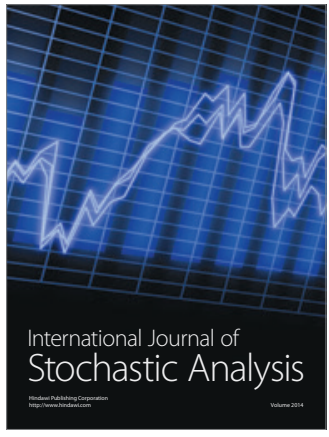

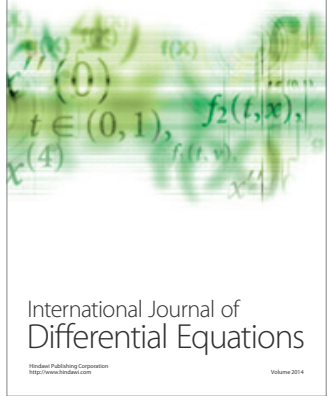
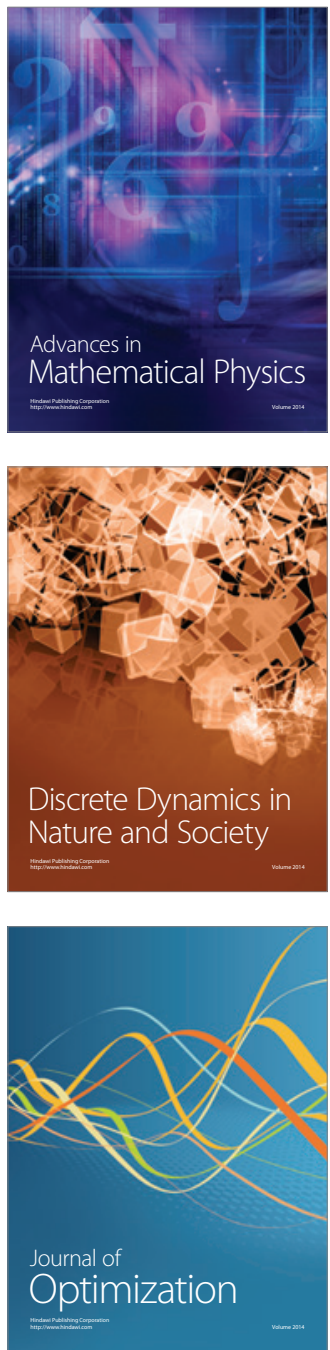